\title{
A biologically based model for recognition of 2-D occluded patterns
}

Mohammad Saifullah, Christian Balkenius and Arne J önsson

The self-archived postprint version of this journal article is available at Linköping University Institutional Repository (DiVA):

http:/ / urn.kb.se/ resolve?urn=urn:nbn:se:liu:diva-110529

N.B.: When citing this work, cite the original publication.

The original publication is available at www.springerlink.com:

Saifullah, M., Balkenius, C., J önsson, A., (2014), A biologically based model for recognition of 2-D occluded patterns, Cognitive Processing, 15(1), 13-28.

https:/ / doi.org/ 10.1007/ s10339-013-0578-9

Original publication available at:

https:// doi.org/ 10.1007/ s10339-013-0578-9

Copyright: Springer

http:/ / www.springer.com/gp/products/journals 


\title{
A Biologically-Inspired Model for Recognition of Occluded 2-D Patterns
}

\begin{abstract}
In this work we present a biologically-inspired model for recognition of occluded patterns. The general architecture of the model is based on the two visual information processing pathways of the human visual system, i.e., the ventral and the dorsal pathways. An eye tracking study was conducted to investigate the human behaviours for the task of occluded pattern recognition and an information processing strategy was devised for the task. The behavioural results from the eye-tracking study support an interactive processing approach preceded by a rapid recognition process. Furthermore, the results underline the importance of handling occluded patterns or noise in the recognition process.
\end{abstract}

The proposed model has a hierarchical structure and consists of three parallel processing channels: the Main channel, the Spatial channel and the Direct channel. The main channel learns invariant representations of the input patterns by extracting local edge features from the input. This channel is responsible for the recognition of input patterns but is limited to process one pattern at a time. The Direct channel represents the biologically based direct connection from the lower to the higher processing level in the human visual cortex. It performs fast processing and computes the top-down pattern specific cues to modulate processing in the other two channels. The Spatial channel mimics the Dorsal pathway of the visual cortex. It generates a combined saliency map of the input patterns and, later, segments the part of the map representing the occluded pattern. The lateral interaction of the Main and the Spatial channels at appropriate processing levels and top-down, pattern specific, modulation of the these two channels by the Direct channel, strengthen the locations and features representing the occluded pattern. Consequently, occluded patterns become focus of attention in the ventral channel and also the pattern selected for further processing along this channel for final recognition.

The core part of this work is on the segmentation of that part of the saliency map which represents the occluded pattern in the stimulus. This segmentation process is based on our hypothesis that the Dorsal pathway, in addition to encoding spatial properties, encodes the shape representations of the patterns as well. In the Spatial channel of the model, the segmentation process is done as an interactive process. Such that bottom-up image based cues and top-down pattern specific cues interact in small processing steps, and result in an incremental development of a segmented map for occluded patterns. This segmented map encodes spatial as well as shape information of the respective pattern in the input. Simulation results support the presented hypothesis as well as effectiveness of the model in providing a biologically-inspired solution to the problem of occluded pattern recognition. Furthermore, the behaviour of the model is in accordance with known human behaviour on occluded patterns.

Keywords: Vision, Occluded Patterns, Neural Network Model, Interactive Network, Biologically-inspired model, Interactive Process, Attention, Segmentation and Recognition.

\section{Introduction:}

Human pattern recognition is superior to present day artificial pattern recognition systems. Humans have the ability to recognize patterns with large variation of position, scale, illumination, occlusion etc. Occlusion is one such factor which renders pattern recognition a challenging problem for artificial systems. Occlusion occurs when a pattern is overlapped by another pattern or noise. It is very common in the real, unconditional $3 \mathrm{D}$ environment, that objects appear with a number of other objects and that they occlude each other when viewed from a certain angle.

Occlusion mainly causes two problems for otherwise efficient pattern recognition systems and thus makes recognition a hard task. Firstly, the system cannot get complete information (features) of those parts of a pattern which are occluded by another pattern. Secondly, the system receives features of both the occluded pattern as well as the occluding pattern, i.e., features from more than one pattern appear in a single view. Recognition of overlapped patterns involves two fundamental issues in the field of computational vision: Segmentation and Recognition. Both in human vision [1][2][3] and in computer vision [4] these two processes are considered as highly interdependent. In a given pattern recognition approach sequential ordering of these two processes is important but varies across approaches. In fact, the order of these two processes in the human visual system is still unsolved and widely debated. Moreover, computational approaches for pattern recognition can be categorized on the basis of this order.

Traditional theories of visual information processing [5][6][7][8] suggest a bottom-up 'segmentation followed by recognition' approach. According to this approach only stimulus based cues, e.g., intensity, continuity of edges, junctions etc., are used to segment the input image into different conceptual regions. Each of the segmented regions is supposed to represent a distinct pattern. Once segmentation is completed the recognition process is carried out on each segmented region. Since this approach does not utilize the class specific top-down cues for segmentation it does not use representations of object knowledge in the segmentation process. Although very efficient, models based on the bottom-up approach have limitations when well-defined stimulus cues are absent. Moreover, most of the patterns are non-homogenous regarding their colour, intensity, etc., and require prior knowledge of pattern class. The work of Gestalt psychologists, particularly [9] and [10] related to finding the 'rules' for the visual system to distinguish between figure and background further supports the bottom-up approach. According to their work image based cues, like, symmetry, convexity, area, etc., are used by the visual system for figure-ground segmentation. 
Another proposed approach to the recognition-segmentation process is top-down 'Recognition followed by segmentation'. In this approach, contrary to the bottom-up approach, as a first step, patterns are identified and, in the next step, top-down cues utilize stored pattern representations for segmentation. The main proponents of this approach are Peterson and colleagues [11][12][13][14] who on the basis of their studies proposed that familiarity plays an important role in segmentation of patterns. They further proposed a 'pre-figural' pattern recognition process before the beginning of any segmentation in a visual system. Recently this approach has achieved much attention among computer vision researchers [15][16][17]. Due to the large variation in shape of patterns of a given class, top-down processes only are not enough to accurately segment patterns in a given image.

There is a third approach which combines the bottom-up and the top-down approach also known as the interactive processing approach. In this approach both segmentation and recognition processes proceed in parallel in small incremental steps and through mutual interaction. Both processes depend on each other and thus facilitate each other. This approach is also based on evidence from human vision studies (as is the topdown approach). But, its proponents reject the top-down approach with an argument that 'If we assume that the goal of sensory segmentation is to provide the correct inputs to object representations, then how can object representations influence segmentation prior to receiving inputs from the earlier segmentation processes?' (1997, p.1293) [18]. In the interactive processing approach the traditional hierarchical structure of information processing remains intact but different levels of processing interact with each other and perform constraint satisfaction style processing. The partial processing results from the lower levels are propagated to the higher levels and then sent back after partial processing. Many studies in cognitive psychology and psychophysics [19][20][21][22] support the interactive processing approach.

In this paper, we introduce a biologically-inspired model for the recognition of 2-D occluded patterns. In this model the segmentation-recognition problem is considered an extended-interactive processing approach, which is a combination of the interactive and the top-down approach.

The rest of this paper is organized as follows: Section 2 discusses background and related work. Section 3 presents a behavioural study on the occluded patterns and its conclusions by using data derived from the experiments. Section 4 presents the method and describes the approach and the model based on the approach. Section 5 describes the simulation and results. In this section the experimental set-up and choice of data set will be explained and then the simulation results will be presented. Section 6 contains discussion and conclusions of the paper.

\section{Related Work and Background:}

In this section an overview of work related to this paper, which also constitute its background, will be presented:

One of the earlier works in this regard was Marr's model [7] of visual processing, which is based on the bottom-up approach. The model is divided into four stages, from bottom-to-top: raw primal sketch, full primal sketch, $21 \frac{1}{2} \mathrm{D}$ primal sketch and 3D model. At the raw primal sketch stage intensity changes are detected, at the full primal sketch stage location of intensity changes are interpreted as contours and boundaries, at the $2 \frac{1}{2} \mathrm{D}$ primal stage local depths and surface orientation is made explicit, and finally the 3D stage combine all information to build a 3D representation of the world. However, in this model the object recognition stage does not provide any class specific information for grouping the features from the 2-D raw primal sketch to the full primal sketch.

Ullman proposed a model-based object recognition system [23] which used high level object specific knowledge to search and locate the features. But his model does not use any stored object knowledge for segmentation in the earlier stages of processing.

Mozer and colleagues [24][25] proposed a neural network model, Multiple-object Adaptive Grouping of Image Components (MAGIC), to segment overlapped patterns. This model is based on the principle of Parallel Distributed Processing (PDP). The hidden layer of this model gets input from the feature representation layer and encodes useful feature conjunctions that facilitate segmentation of boundaries through learning. The significant thing of this model is that the network did not use hard coded, built in, heuristics; rather it discovers the grouping principles through learning. However, their model is basically a bottom-up approach as it does not utilize any high level pattern specific knowledge to guide the segmentation process.

In their Preconscious oscillations theory Rock [26] explained how high level cues influence the bottom-up segmentation process. According to this theory when someone views a stimulus a region of the given stimulus is precociously selected and considered as a figure and then matched against stored representations. If a match occurs than this region represents a figure in the stimulus otherwise the selection is shifted to other regions. This theory also supports bottom-up serial processing as segmentation of parts of the preconscious figure occurs before matching for recognition.

Peterson and colleagues [13][12][1] presented a theoretical 'pre-figural' model, and proposed that there are two types of cues for image segmentation. The first type of cue is a stimulus based 'Gestalt' cue and the second type of cue is an early orientation-dependent, high level object representation process. According to this model the object recognition process determines two sets of parts by operating on both sides of the luminance contour in a given stimulus. If one of these sets matches the stored object representation in the visual memory then this representation provides top-down cues to the low level segmentation process. In this model the second cue is computed before completion of the first cue which is based on the segmentation process. This model clearly suggests that the object recognition process precedes the segmentation process. 
Vecera and O'Reilly [2] presented a computational model of figure-ground organization that is based on the Parallel Distributed Processing (PDP) model [27][28] of information processing. In their model they implemented the more general principles of graded, interactive processing. Their model is a hierarchical model with three processing layers. The first layer extracts oriented edges from the input images. The second layer called the figure unit, groups the features representing the figure and the third layer is the output layer which represents the individual objects. These layers interact with each other through excitatory connections. The second layer, the figure unit layer, gets bottom-up (image information) as well as top-down (object representation) information and thus the model performs processing under these two constraints. An additional sparsity constraint in the form of a KWTA (K-Winners-Take-All) mechanism was introduced which improves the results and reduce the simulation time; moreover, it matches with the biologically plausible inhibition in the visual system. The model performs equally well for both ambiguous and unambiguous images. In case of unambiguous images an extra layer, which represents the disparity in the input, is added. This layer receives input directly from the image and connects it to the figure unit layer with one-to-one connectivity.

In computer vision, most work on the segmentation recognition problem is based on the bottom-up approach. In these approaches both region based properties and boundary properties are used for segmentation. For example [29] uses the colour, [30] uses the intensity and [31] utilizes continuity of body contours as bottom-up cues for segmentation.

Recently there has been an increased interest for using object related knowledge for figure-ground segmentation. Borenstein and Ulman [15] represented the images into small fragments together with labels of object and background labelling. Given an image containing a known object class, these fragments were used in a jigsaw-puzzle fashion to fit the image which results in a consistent mapping of object-background labelling. They produced impressive results on the segmentation of side viewed horses images. In their model they did not recognize the segmented part which might result from segmentation of background clutter that matches the object part randomly. In later work [32], they extended and improved the segmentation results by combing top-down and bottom-up processing cues. For bottom-up processing they used the uniformity criteria to create a hierarchy of segmented regions.

Another significant work in computer vision that considers segmentation and recognition as intertwined tightly coupled processes that collaborate for a common goal is by Leibe and colleagues [33]. The main part of their approach is on learning highly flexible shape representations of objects in a probabilistic extension of the Generalized Hough Transform. These representations are used to segment the pixels representing the objects in a given image. The segmentation results in turn improve the recognition by providing to the system an improved set of pixels of that specific object, with little misleading background pixels. Their proposed system is demonstrated to be applicable to both rigid as well as articulated objects and achieve competitive performance with comparatively smaller training data.

\section{Eye-Tracking for Occluded Patterns Recognition:}

In order to investigate and understand human behaviour for recognition of 2D-occluded patterns an eye tracking study was conducted. The main emphasis of this investigation was to analyse the strategy that is employed by humans for occluded pattern recognition.

It is common knowledge that humans perform a number of saccades on a stimulus before recognition, especially when the pattern is relatively difficult to recognize. There are evidences from human vision studies [34][35][36] that the visual system can perform rapid categorization in just fractions of a second with little time for any eye movements or shift of attention. This early rapid categorization or immediate recognition involves only feed-forward connectivity in the visual system. Another important illustration is the Kanizsa-Bergman Display [37], which implies that it is easier for humans to recognize patterns occluded by other patterns than to recognize patterns with missing parts. These issues were considered in the study as well as their effect on the overall strategy..

The main questions to investigate were: What role does immediate pattern recognition has for recognition of occluded patterns; what is the role of occluding patterns (or noise) for recognition occluded patterns; and which approach does humans adopt to recognize occluded patterns. In this section a brief description of the method and the results of the study are presented.

\subsection{Method:}

Subjects: Five Linkoping University staff and students were recruited as human subjects with corrected or corrected-to-normal vision. Subjects included four males and one female aged between 21- and 40 years. All subjects were well conversant with the English language and had the proven ability to easily recognize English alphabets and digits. The subjects had never seen the stimuli before and were naive to the purpose of the experiment. 


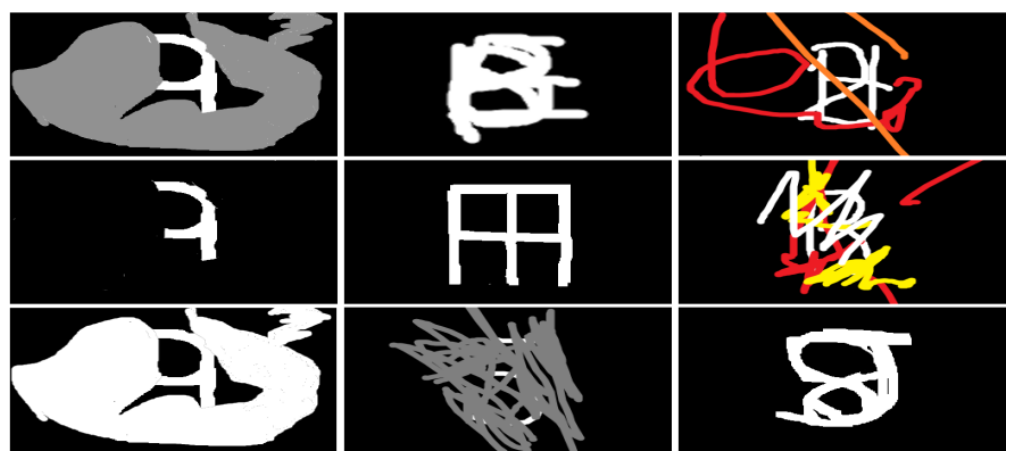

Fig. 1. Some of the stimuli used in the eye tracking experiment.

Stimuli: In total twenty four stimuli were used in the experiment. Some of the stimuli are depicted in Figure 1. The stimuli were made up of patterns containing both capital English letters and digits. Each stimulus was developed by drawing a pattern manually and then random noise or another pattern was superimposed on that. All occluding patterns (the main pattern of interest) have the same gray scale value (White) whereas occluding noise or pattern may have different intensity values. Moreover, some of the stimulus had three variants (Figure 1, first column), such that, the three stimuli had the same occluded and occluding patterns but the intensity values of the occluding pattern where different for each. In the first stimulus the occluded and occluding patterns had different intensity values from each other as well as from the background. In the second stimulus the two patterns had the same intensity value and the background had a different intensity value. In third stimulus the occluding pattern and the background had similar intensity value and hence the pattern looks like a pattern with missing parts.

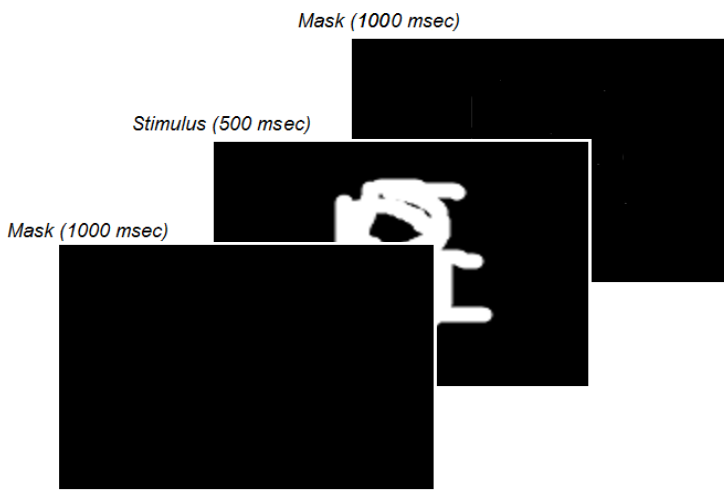

(a)

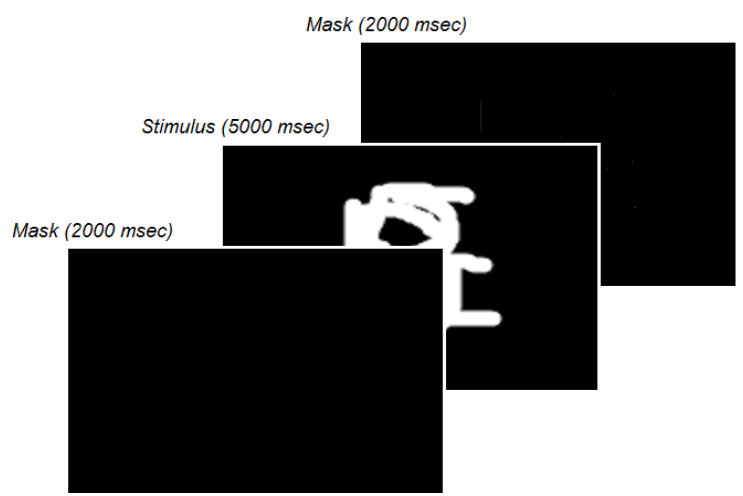

(b)

Fig. 2. Two time schemes for Eye tracking experiment procedures (a) Stimuli presented for a brief 500 msec followed by a mask for 1000 msec. (b) Stimuli presented for a brief 5000 msec followed by a mask for $2000 \mathrm{msec}$.

\section{Procedure and Task:}

We used the iView X eye tracking system [] to record eye gaze position during the course of the experiment. Eye position was sampled at a rate of $50 \mathrm{~Hz}$. Both pupil and corneal reflection from the right eye were used to determine the gaze position on an image. Stimuli were presented on a 1920x1080 pixels LCD monitor and subjects were seated in front of it on a comfortable chair.

The same sets of stimuli were presented to the subjects with two different time schemes. In the first scheme each of the stimuli was flashed to the subjects for a short duration of only $500 \mathrm{msec}$. After presentation of each stimulus a mask is presented for another $1000 \mathrm{msec}$. The task for the subjects was to identify and speak out the name of the pattern in the stimulus as soon as possible or at least before disappearance of the stimulus. The responses of the subjects were recorded both manually as well as electronically for later analysis.

In the second scheme each of the stimuli was presented to the subjects for $5000 \mathrm{~ms}$ followed by a mask for another $2000 \mathrm{msec}$. During the presentation of each stimulus a ticking sound was played with equal intervals of $1000 \mathrm{msec}$, such that five ticks could be heard during the presentation span of each stimulus. The task for the subjects was to speak out the name of the occluded pattern (in a stimulus) after each tick sound, if they recognize or have got a slight idea of it. Meanwhile, subjects can ignore the tick sound if they have no idea of the identity of the pattern at that instance. Furthermore, subjects were asked to find just one pattern and continue recognizing it (enhance the confidence level) for the whole presentation time of that stimulus. If they change their mind about the identity of the pattern during the stimulus life span they must speak out the new identity. Before actually conducting the experiments each subject was properly rehearsed and intimated with the experiment equipment and procedure, so that he/she could properly understand the rules of the game. Moreover, the subjects were informed that patterns of 
interest always appear with a specific intensity value (in this study in white colour) whereas noise may have different intensity values than that of the patterns of interest.

3.2 Analysis and Discussion: On the basis of the eye-tracking experiments and analysis of the results we made some conclusions, which are presented in the following:
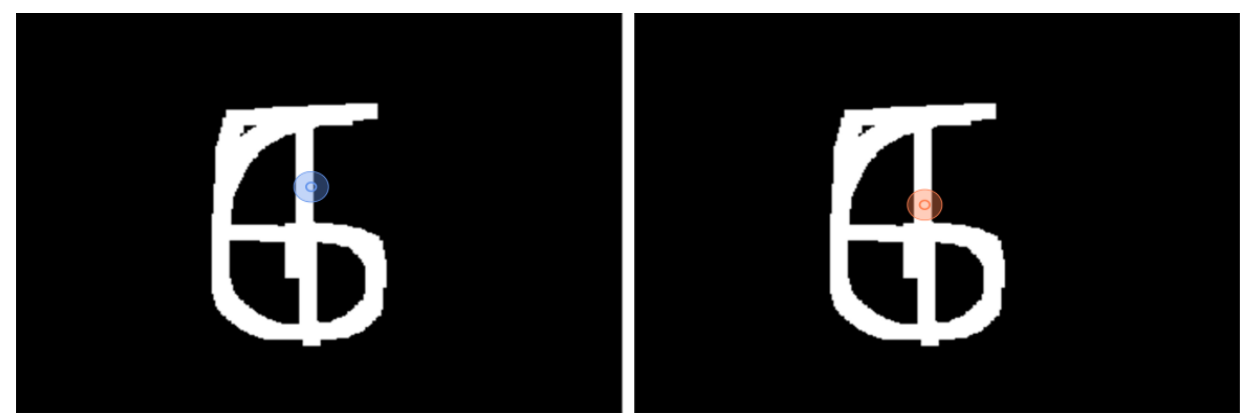

Fig. 3. Scan path of two of the subjects when a stimulus is presented for $500 \mathrm{msec}$. The circular marks on each instance of the stimulus represent the scan path of the subjects overlaid on the stimulus. The scan path shows gaze positions and eye events of the selected trial data set plotted on the stimulus image. It is clear from the scan path that the subjects focused on a single point as they did not have enough time to move their eyes.

i. The early/immediate recognition process compute top-down cues and influence subsequent processing.

The results indicate that subjects can guess the identity of the occluded pattern in a given stimulus very early and then analyse the stimulus for verification of this guess. Usually this early guess tends to be highly influential (and beneficial) in setting the trends for subsequent processing, i.e., modulating the bottom-up processing in favour of a particular pattern class. However, influence of the earlier top-down cues can be overridden if bottom-up cues contain some strong features specific to some other pattern. Now consider Figure 3, which shows the scan path of two of the subjects' eye-tracking experiment when the stimulus was flashed for $500 \mathrm{msec}$. It can be observed that the subjects do not have enough time to change their gaze. Nevertheless, the subject can guess the pattern with some accuracy even in this short duration of time. It can be noted that this initial pattern recognition ability is, most probably, based on a template matching process (as the subjects do not change their gaze and the time is too short). It is also quite evident from later experiments that subjects tend to follow the strokes of the pattern they guess in the beginning; which implies that this initial guess influences the subsequent visual processing as top-down cues.
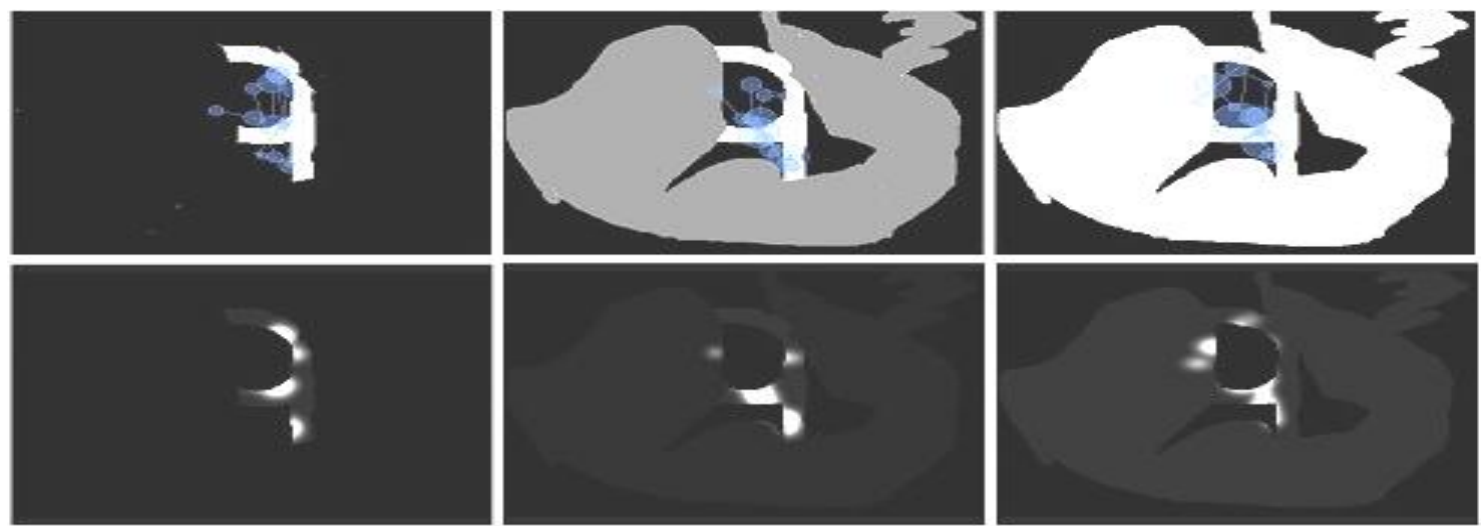

Fig. 4 . Scan path (top row) and focus map (bottom row) of a subject eye tracking data overlaid on the stimuli. Stimuli are three variant of a single stimulus. It can be seen that in case of patterns with occluding parts (second and third column from left) the subject tend to move gaze over the occluding pattern in search of occluded parts. On the other hand, in case of patterns with missing parts (first column from left), no such effort is made.

\section{ii. Occluding patterns facilitate recognition of occluded patterns}

Statistics of the results were in-line with the general perception that humans can recognize patterns occluded with other patterns more accurately than the patterns with missing parts. The subjects' eye movement behaviour gives some insight into the question of how occluding patterns help recognition of occluded patterns. Figure 4 shows the scan path and focus map of a subject's eye tracking data overlaid on the stimulus image. In the focus map gaze patterns are visualized by altering the transparency of the stimulus display based on the amount of the attention received. It is also worth mentioning that all subjects show very similar gaze behaviour on the recognition tasks. It can be seen that the subjects tend to explore 
the parts of the pattern occluded behind the other pattern or noise. It seems that occluding patterns give the viewers some kind of perceptual cues that occluded pattern stretch behind the occluding pattern. This implies that occluding patterns play an important role and facilitate pattern recognition of occluded patterns.
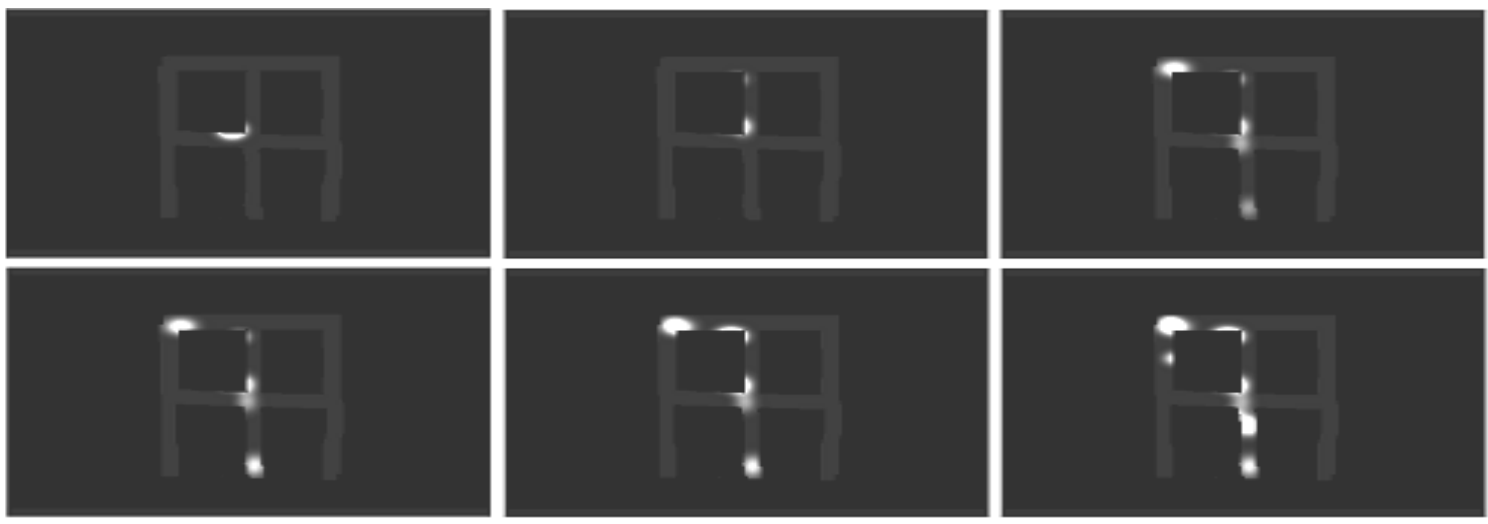

Fig. 5. Focus maps of a subject's eye-tracking data overlaid on a stimulus at six sequential time steps, i.e., first image is for $\mathrm{t}=0.5 \mathrm{msec}$ and later each image after duration of $1 \mathrm{msec}$. The order of the images in the figure is: from left top row and then bottom row.

iii. The strategy to recognize occluded patterns is extended based on interactive processing.

The recorded eye tracking data endorse the interactive strategy for recognition of occluded patterns. All of the subjects show a similar behaviour on the occluded pattern recognition task. As a general strategy they made a guess at the beginning of each task and then on the basis of this guess they follow the strokes of the guessed patterns. If during the task they get confused about the identity of the pattern, by finding features related to some other pattern or lack of features that support the present guessed pattern, they tend to follow the new guess pattern. For example, consider Figure 5 which presents a single task. A stimulus is presented to a subject, who makes an initial guess and then soon realizes that it is a wrong guess and changes it. The first image, from left on the top row, presents the focus map for the first $500 \mathrm{msec}$ when the subject guesses that the pattern is an 'A'. At $1000 \mathrm{msec}$ (second image) the subject realizes that the guess was a mistake as he/she found that the feature did not match the guessed pattern, however he/she did not respond because of confusion. At $2000 \mathrm{msec}$ (third image) he/she considers the pattern as ' $\mathrm{T}$ ' and follows the strokes of the pattern ' $T$ ' under the influence of top-down pattern knowledge. The rest of the time the subject recognizes the pattern as ' $T$ ' as the top-down and bottom-up cues agrees, and he/she founds the features of the pattern ' $\mathrm{T}$ ' in the stimulus.

This strategy clearly endorses the interactive processing approach that partial bottom-up results are sent to the higher levels for recognition and that top-down cues are sent back for further processing and verification. This strategy is identical to the interactive strategy suggested by Vecera and Farah [18]. But if we take into account the early recognition process then it can be said that the general strategy adopted by humans is an extended interactive approach such that it involves a 'pre-figural' recognition process followed by an interactive process.

On the basis of this study we concluded that humans make an early guess at the beginning of a recognition process and then use this guess as a top-down cue to analyse the stimulus for validation of the guess. The role of the occluding pattern is important in recognition and it gives humans a feeling that the required pattern is extended under the occluding pattern. On the basis of these results and previous knowledge about the architecture of the human visual system we present a model for recognition of occluded patterns.

\section{Overview of the Approach:}

The approach used in this work is inspired by the two parallel and recurrent processing pathways of the human visual system, i.e., the ventral and the dorsal pathways. This is related to and a succession of earlier work on the visual attention model in which attention emerges as a result of interaction between the two processing pathways which selects a specific location or pattern for further processing [38][39]. In the present work the input information will be processed along three channels which represent the ventral pathway, dorsal pathway and direct connections from lower to higher level of processing respectively.

Among the main features of the model's architecture are: the principle of hierarchical sequences of transformations to produce spatially invariant representations; increasing receptive field sizes along the hierarchy and; development of increasingly complex and invariant representations along the hierarchy from lower to higher levels. The adjacent levels of the hierarchy are connected to each other through bidirectional connections, which not only provide a biologically plausible propagation of errors, but also facilitate a constraint satisfaction style of recurrent processing between the levels. The ventral channel of this model encodes the shape of the patterns by extracting local edge features using Gabor filters. These features are systematically collapsed along the hierarchy to develop invariant shape representations of the patterns. This pathway has the capacity to process one pattern at a given time. Direct connections from the lowest to higher levels are also included in the model, which has biological plausibility. This provides fast top-down cues, on the basis of template matching, to modulate the processing pathways. The dorsal channel of this 
model encodes the spatial as well as somewhat the shape information of the input patterns. At the lowest level this pathway an activation-strength based saliency map of the input stimulus is developed. At the subsequent level this map is segmented for occluded patterns present in the map. The segmentation is performed under the influence of top-down pattern knowledge. The general strategy is that the dorsal pathway, with the help of a segmented map of the pattern, modulates processing in the ventral pathway in favour of that pattern. As a result of this modulation and KWTA constrains within the levels, the pattern corresponding to the segmented map in the dorsal pathway is strengthened and selected for further processing along the ventral pathway.

The main thrust of this work is on sequential segmentation of the saliency map for each of the overlapped patterns within the model's dorsal pathway. The Segmentation process is based on our hypothesis that the dorsal pathway of the visual system encodes the shape knowledge, in addition to spatial information of the patterns, and then uses this information to perform segmentation of the overlapped patterns. There are evidences from neuroscience [40][41] that neurons in the lateral intraparietal cortex (LIP) part of the dorsal pathway show some selectivity for shapes of the patterns and hence suggest the possibility of encoding shape information along this pathway. Unlike traditional visual information processing theories, which consider segmentation and recognition as two independent processes, the dorsal pathway of this model is implemented as an interactive process. In this approach a patch of the bottom-up saliency map is selected and identified as part of a specific pattern by matching it with the stored templates and corresponding identified pattern class activated at the higher level. This higher level perception then sends back top-down signals to guide the segmentation process. This interactive process of segmentation proceeds in small steps and develops a map that encodes the spatial location as well as shape information of the occluded patterns.

The proposed model is realized by developing an interactive (recurrent, bidirectional) neural network.

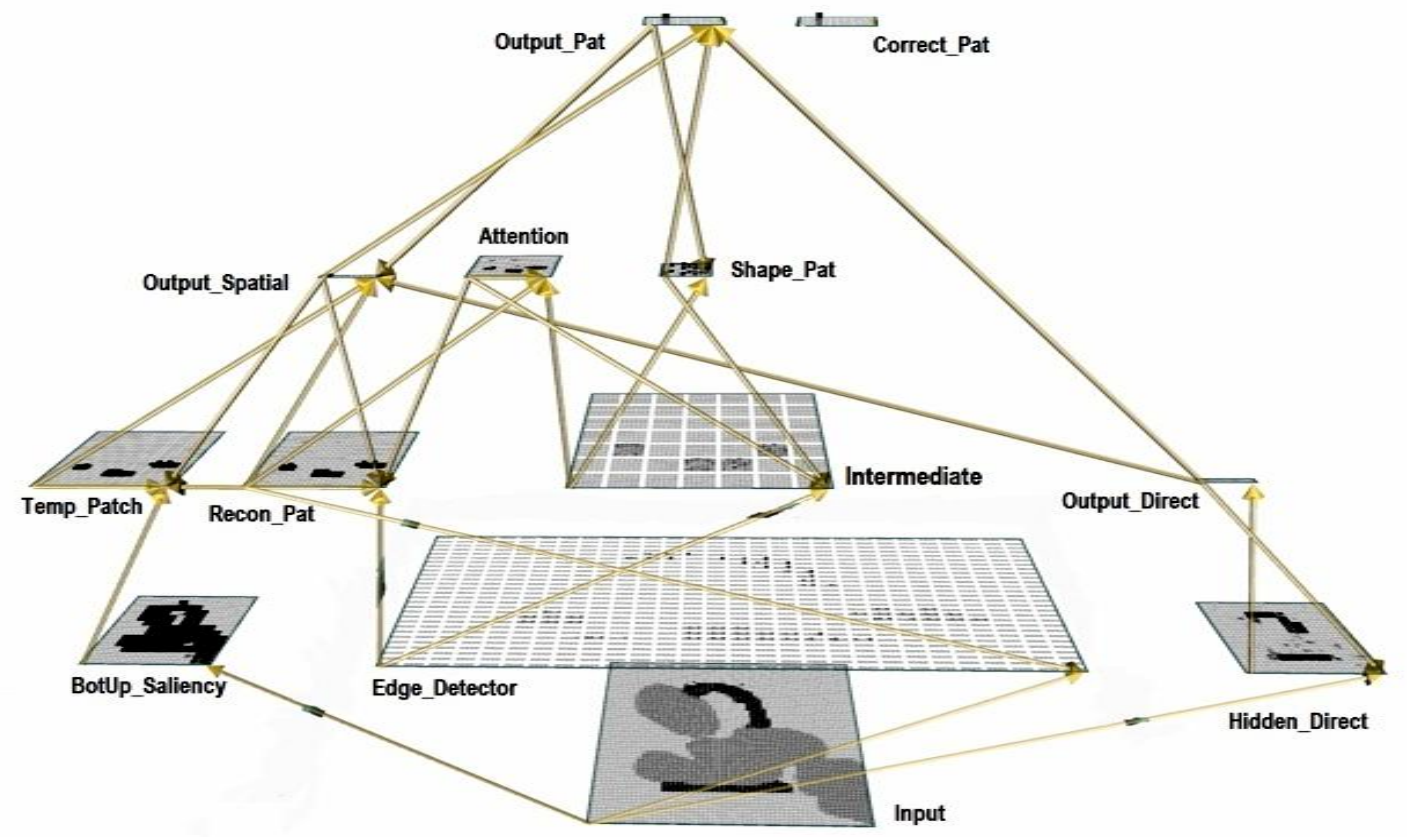

Fig.6. The neural network used in the simulation.

4.1 Model: The proposed model is a hierarchical multi-layered neural network, as shown in the Figure 6, and illustrated in the schematic diagram (Figure xxx). It is composed of three channels (sub-networks): Main channel, Direct channel and Spatial channel. In the following a brief description of each of the channel is presented.

Main channel: The primary task of this channel is to recognize a given pattern in a scale, position and distortion invariant manners and thus it performs the role of the ventral channel in this model. This channel learns invariant representations of the input patterns while processing information along its hierarchical structure.

The first layer of this pathway is named Edge_Rep which represents the V1 or the primary visual cortex area. It is designed to detect oriented edges like biologically-inspired features from the input image. The units of this layer are arranged into groups such that each group processes a specific part of the input image. All units within a group of the Edge_Rep layer detect similar oriented edge features from the same spatial location, but for different orientation. Gabor filter based edge detectors are used for extracting these features. 
The next layer along the hierarchy is the Intermediate_Rep layer which represents the V2 area of the visual cortex. It is also a multi group layer. Units of this layer have larger receptive fields than that of Edge_Rep units. Units in this layer process the information encoded by the Edge_Rep units. During learning, this layer develops more complex and invariant feature representations. Since the units of this layer are divided into groups the topographic representation of the input is still rather vague.

The next layer along the hierarchy is the Shape_Rep layer which represents the higher processing areas. This layer is homogeneous in the sense that it is not subdivided into unit groups. Units in this layer have a very large receptive field which encompasses the whole of the input, i.e., each unit of this layer gets input from all of the units in previous layers. This layer builds a complex and invariant representation of individual patterns. Since all units of this layer share a single receptive field, topographic information is completely lost at this layer.

The last layer of the model is the Main_Output layer which is an output layer. Each unit of this layer represents a unique pattern class; therefore, the number of units in the output layer is equal to the number of classes for which the network is trained. Error-driven learning in combination with Hebbian learning is used to assign all representations of a specific class of patterns at the Shape_Rep/IT layer to its class-representing unit at the output layer.

Direct channel: This channel is a simple three layered network and accounts for the direct connections from the lower to the higher processing areas of the visual cortex. Its main task is to immediately classify the input patterns and provide top-down pattern specific cues to modulate the processing in other networks. This network stores (learns) shape templates of the input pattern classes and predicts identity of a pattern on the basis of close matches with the stored representations.

The three layers of this network are Input, Hidden_Direct and Direct_Output. The Input layer is the same as for the Main channel. It is shared by all three channels. The Hidden_Direct layer, the hidden layer of the network, is connected to the Input layer through bottom-up connections. This layer is smaller in size than the input layer, such that each unit of this layer receives input from a small group of the Input layer which constitute the receptive field of that unit. This connectivity pattern between two layers helps to reduce (to some extent) shape distortion as well as position and size variations among patterns of the same class. This stage processes information solely on the basis of its connectivity patterns and there is no learning connection between these two layers. The next processing layer of the network is the Direct_Output layer, which is connected to the previous layer through bidirectional connections. Each unit of this layer represents one distinct class. The templates generated at the Hidden_Direct layer are associated with their corresponding class of the Direct_Output layer through learning.

Spatial channel: The main functionality of this channel is to assist the ventral channel processing one pattern at a time. In this study the task of this channel is to segment the occluded pattern from the occluding patterns. This channel encodes spatial information of the input patterns. It also learns the shape of the input pattern and uses this information to guide the segmentation of the patterns. This channel interacts with the Main channel at appropriate levels and modulates it in favour of specific locations and features. Since this channel encodes the spatial representation of the patterns, which is topographic in nature, it must interact with those stages of the Main channel which keep the topographic representation of the input, in addition to pattern specific information.

The first processing stage in this channel is the BotUp_Saliency layer, which draws its input directly from the Input layer and develops a bottomup saliency map of the input. Its size, functionality and connectivity pattern with the Input layer is the same as that of the Hidden_Direct layer with the Input layer. The only difference between the two layers is the threshold values for the units' activations. The Hidden_Direct layer generates the templates of the occluded parts and other patterns having the same intensity value, whereas the BotUp_Saliency layer generates the saliency map for all input intensity values.

Next along the processing hierarchy is the Temp_Patch layer, which has the same size as the BotUp_Saliency layer and is connected to it through a one-to-one connectivity pattern. Moreover, each unit of this layer is connected to its eight close neighbours through self-connection. This layer allows only a very small number of units to be active at a given time, furthermore, the self-connectivity forces the active units to appear in the form of one or two groups. Additionally, a mechanism of 'accommodation' is employed on the units of this specific layer. This mechanism causes a unit that is active for a while to get tired and die down for a short period of time, thereby giving way to other competing units to become active. In this way active group(s) of units seems to be moving in the previous layer.

The next layer is Recon_Pat, which has the same size as the last two layers, described above, and is connected to the Temp_Patch layer through a one-to-one connectivity pattern. This layer has the large K value for KWTA comparing to the previous layer. In this layer an additional mechanism of 'hysteresis' is used, which causes active units to remain active for a longer period of time even after removal of their excitatory input. In this way, this layer keeps the traces of the patches which get active.

The next layer, the Attention layer, is connected laterally to the Temp_Patch and Intermediate_Rep layers. This layer generates an attention map on the basis of input activations from the Recon_Pat and Intermediate_Rep layers. The influence of the Recon_Pat layer is far greater than that of the Intermediate_Rep layer. The attention map modulates the units of the Intermediate_Rep layer in favour of a specific pattern and facilitates the main channel to activate representations that belong to a single pattern.

The Spatial_Output layer is the highest processing layer of the spatial channel. It associates template like spatial as well as shape representations of the input patterns that are developed at the Temp_Patch and Recon_Pat layers, to a specific class. This layer receives bottom-up cues from the lower processing areas in the same channel as well as top-down cues from the Direct channel, and in return guides the segmentation processes interactively. 
Table 1. Specifications of the network used in simulation.

\begin{tabular}{|c|c|c|c|c|c|}
\hline Layer Name & Type & Unit Geometry & $\begin{array}{c}\text { Group } \\
\text { Geometry }\end{array}$ & $\begin{array}{c}\text { Receptive } \\
\text { Field of a unit }\end{array}$ & $\begin{array}{c}\text { Receptive } \\
\text { Field Overlap }\end{array}$ \\
\hline Input & Input & $60 \times 60$ & - & - & \\
\hline Edge_Detector & Hidden & $4 \times 1$ & $28 \times 28$ & $4 \times 4$ & $2 \times 2$ \\
\hline Conjunction & Hidden & $6 \times 6$ & $7 \times 7$ & $16 \times 4$ & - \\
\hline Shape & Hidden & $8 \times 8$ & - & Whole layer & - \\
\hline Hidden_Direct & Hidden & $26 \times 26$ & - & $5 \times 5$ & $\begin{array}{c}5 \times 5 \text { and } 1 \times 1 \\
\text { Alternatively }\end{array}$ \\
\hline BotUp_Saliency & Hidden & $26 \times 26$ & - & $5 \times 5$ & $\begin{array}{l}5 \times 5 \text { and } 1 \times 1 \\
\text { Alternatively }\end{array}$ \\
\hline Tem_Pat & Hidden & $26 \times 26$ & - & $1 \times 1$ & - \\
\hline Recon_Pat & Hidden & $26 \times 26$ & - & $1 \times 1$ & - \\
\hline Attention & Hidden & $14 \times 14$ & - & $1 \times 1$ & - \\
\hline Output_Pat & $\begin{array}{c}\text { Target } \\
\text { /Hidden }\end{array}$ & $10 \mathrm{x} 1$ & - & Whole layer & - \\
\hline Direct_Output & $\begin{array}{c}\text { Target } \\
\text { /Hidden }\end{array}$ & $10 \times 1$ & - & Whole layer & - \\
\hline Spatial_Output & $\begin{array}{c}\text { Target } \\
\text { /Hidden }\end{array}$ & $10 \mathrm{x} 1$ & - & Whole layer & - \\
\hline
\end{tabular}

4.2 Network Algorithm: In this study simulations were carried out by using the Leabra (Local, Error-driven and Associative, Biologically Realistic Algorithm) [39] modelling framework which is based on the biologically plausible principals to mimic visual processing. Leabra models can combine supervised error driven learning with unsupervised hebbian learning through a combination of biologically plausible feedback loops, a k-winners-take-all inhibition method, and a two phase expectation/outcome learning process. The Leabra algorithm is implemented in Emergent [42] a comprehensive neural network simulation environment for neural network modelling.

Activation Function for the Basic Unit in Leabra: In Leabra, the basic unit is a point approximation of a biological neuron. All necessary computations regarding the basic unit are made according to a formula that is derived by analysing the electrophysiological properties of a biological neuron. An important parameter of a biological neuron is its membrane potential, which can be described as a function of all the input to the neuron through its dendrites. The value of the membrane potential, together with a threshold, is responsible for determining the output of the neuron. Leabra models the output of a neuron by using the thresholded sigmoidal function of the membrane potential (equation 1):

$$
y_{j}=\frac{\gamma\left[V_{m}-\theta\right]_{+}}{\gamma\left[V_{m}-\theta\right]_{+}+1}
$$

Where, $\gamma=$ gain, $V_{m}=$ membrane potential, $\Theta=$ firing threshold, $[x]_{+}=$positive component of $\mathrm{x}$, otherwise zero

Hebbian / Model Learning: Hebbian learning or Model learning is about developing an internal model of the environment. For model learning, Leabra uses CPCA (Conditional Principal Component Analysis) augmented with weight renormalization and contrast enhancement, which improves the dynamic range of the weights and the selectivity of the units to the strongest correlations in the input. The weight update equation for the Hebbian model learning is given in equation 2:

$$
\begin{gathered}
\Delta w_{i j}=\varepsilon y_{j}\left(x_{i}-w_{i j}\right) \\
=\Delta_{\text {hebb }}
\end{gathered}
$$

Where, $\varepsilon=$ learning rate, $x_{i}=$ activation of sending unit $I, y_{j}=$ activation of receiving unit $j, w_{i j}=$ weight from unit $i$ to unit $j$

K-Winners-Take-All (KWTA): To achieve a sparse distributed representation, Leabra uses a K-Winners-Take-All (kWTA) inhibition function. The KWTA function computes threshold values which allow only the K most active units in a layer to become activated while keeping the remaining weaker units under their firing threshold. The amount of inhibition $g_{i}$, which is provided to the layer or unit group is defined to lie 
somewhere between the inhibition threshold of unit $k+1, g_{i}^{\theta}(k+1)$, which is the amount of inhibition that is required to press unit $k+1$ below its activation threshold, and the inhibition threshold, $g_{i}^{\theta}(k)$, of unit $k$ :

$$
g_{i}=g_{i}^{\theta}(k+1)+q\left(g_{i}^{\theta}(k)-g_{i}^{\theta}(k+1)\right)
$$

Where, $\quad g_{i}^{\theta}(k)=$ inhibition threshold for unit $k, \quad q=$ margin above required level

The combination of hebbian model learning and inhibitory competition leads to a distributed representation of the input patterns, such that units represent the statistically-informative principal features of the input.

Error-driven Task Learning: Model learning learns an internal model of the outside world, but it has limitations when it comes to learning input-output mappings. This makes model learning insufficient for learning a specific task. For this reason, in Leabra, model learning is complemented with error-driven task learning. Error driven learning in Leabra is realized by Contrastive Hebbian learning (CHL) which is an improved form of the biologically plausible GeneRec algorithm [43]. To realize error-driven learning in a biological plausible manner, the network settles in two phases, i.e., expectation (minus) and outcome (plus) phase. The network then computes a simple difference of a presynaptic and postsynaptic activation product across these two phases:

$$
\begin{gathered}
\Delta w_{i j}=\varepsilon\left(x_{i}^{+} y_{j}^{+}-x_{i}^{-} y_{j}^{-}\right) \\
=\Delta_{e r r}
\end{gathered}
$$

Where, $\varepsilon=$ learning rate, $x_{i}=$ activation of sending unit $I, y_{j}=$ activation of receiving unit $j, x^{+}, y^{+}=$act when also output clamped, $x^{-}, y^{-}=$act when only input is clamped

Combined Hebbian and Error-driven Learning: In Leabra, Hebbian learning and error-driven task learning is combined, at each synapse in the network, to obtain the advantages of the two forms of learning. A net weight update of a connection as a result of a linear combination of the two learning methods is:

$$
\Delta w_{i j}=\varepsilon\left[c_{h e b b} \Delta_{h e b b}+\left(1-c_{h e b b}\right) \Delta_{e r r}\right]
$$

Where, $\varepsilon=$ learning rate, $c_{\text {hebb }}=$ proportion of Hebbian learning

The combination of the two forms of learning allows the model to learn the statistical regularities in the input data, and do this in a way that suits the task at hand.

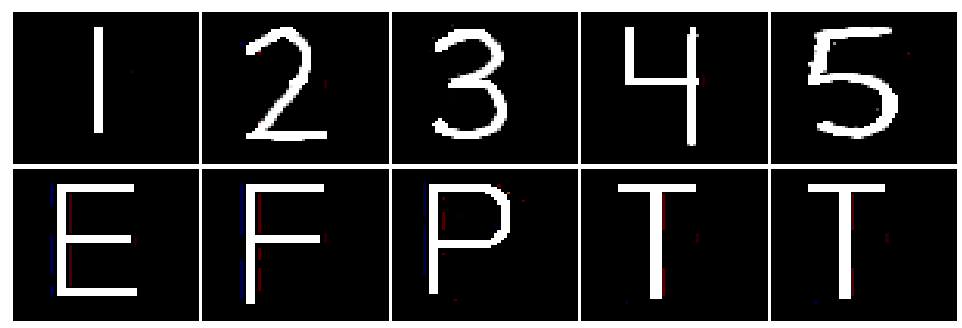

Fig. 7. Images of patterns used for training the network and developing occluded patterns (test stimuli).
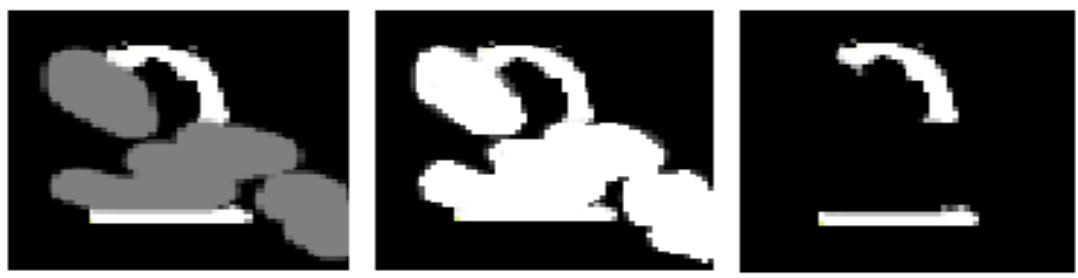

Fig. 8. Stimuli used for testing each of the individual channel and integrated model. All three stimuli are versions of the same pattern. From Left: The first stimulus is a pattern occluded with the noisy pattern having different intensity than the occluded pattern. In the second stimulus occluded and occluding patterns both have same intensity values. The third stimulus is created by removing the noise, such that the pattern becomes the one with missing parts. 


\section{Simulations:}

In this section, first, details about the dataset and training procedure of the model will be described and, later, simulations and results will be presented.

\subsection{Data and Training:}

Dataset: For training ten pattern classes were selected. These patterns are images of five letters of the English alphabet and five digits (Figure. 7). Both machine printed as well as handwritten patterns were used as data set for training. Each of the patterns is shifted two steps in eight directions, where each step is four pixels wide. For testing, stimuli containing occluded patterns were developed (Figure. 8). Each of the test stimuli has three variants: first, the occluding patterns have different intensity values than that of the occluded pattern as well as from background, second both occluding and occluded patterns has the same intensity values, whereas in the third variant the occluding pattern and the background have the same intensity values.

Training and testing: Since all three channels communicate with each other through static connections (not learnable) training of the three network channels can be performed separately, one-by-one, without any specific order.

Training the Main channel: The main channel learns invariant representations of the input pattern classes on the basis of edge features extracted at the lowest level, i.e., the Edge_Rep layer, by using Gabor filters. For learning a mix of hebbian and error-driven learning algorithm was employed. For both bottom-up as well as top-down connection Chebb $=0.002$ was used. The channel was trained for five batches whereas each batch runs until the set stopping criteria of zero-percent-error is achieved.

Training the Direct channel: The Direct channel learns to associate the templates of the patterns, developed at the Hidden_Direct layer, with its Direct_output layer. For this reason a high value of Chebb $(=0.5)$ is used for the connection between the Hidden_Direct and the Direct_Output layer.

Training the Spatial channel: The three layers of this channel, which have learnable connections, learn the templates and associate those with the eight pattern classes represented at the Out_Spatial layer. The Chebb value is the same as for learning the dorsal channel, i.e., 0.5.

5.2 Simulations and Results: In this section the simulation behaviour of the model for the task of occluded pattern recognition will be presented. In the first step, each of the processing channels of the model was simulated standalone, without any influence from the rest of the channels. In the second step, all three channels were combined and the resulting integrated model was simulated on the same task. Each of the simulation trials was run for 400 processing cycles.

\section{Simulation 1: Immediate recognition by the direct channel}

Here we will present the simulation of the Driect channel and will discuss its behaviour. In this simulation the channel worked independently, but its behaviour would remain the same even if combined with the other channels, as it does not receive any input from the other sources except for the Input layer. Three different occluded variants of the same pattern were presented to the model.

\begin{tabular}{|c|c|c|c|c|}
\hline Cycle & Input & Hidden_Direct & Direct_Out & Correct_Out \\
\hline O & & & & \\
\hline 7 & & & & \\
\hline 13 & & & & \\
\hline 100 & & & & \\
\hline 400 & & & & \\
\hline
\end{tabular}

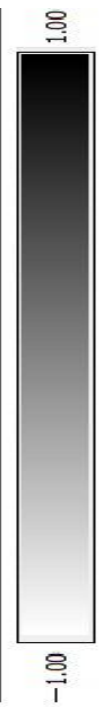


Fig. 9. Snapshots of the activations in the Direct channel's layer for processing cycles when a stimulus containing occluded and occluding pattern having different intensity values are presented to the channel. Each layer is made up of a matrix of units and the activation values of these matrices are shown. Activations in the layers change as the cycle proceeds. In this figure, in the order from left to right, the columns represent: Number of processing cycles (how far computation of the activation has progressed), activations in Input layer, Hidden_Direct layer and Direct_Output layer of the network. Dark colours denote high activation values, light colours low activation.

Occluded and occluding patterns have different intensity values: Figure 9, shows cycle-wise processing results of the channel. The simulation was initiated by clamping a stimulus to the Input layer (cycle: 0 ). The stimulus is composed of pattern '2' occluded by a random noisy pattern which has different intensity value than that of the occluded pattern. It is difficult even for humans to identify the pattern accurately with full confidence. The Hidden_Direct layer generates the template of the occluding pattern only (cycle: 7- 400), by discriminating patterns on the basis of their intensity. The Direct_Output layer shows three active units having different activation values (cycle: 13 - 400I). It means according to this channel the occluding pattern belongs to one of the three pattern classes, represented by the three active units. The high activation value corresponds to high confidence.

\begin{tabular}{|c|c|c|c|c|}
\hline Cycle & Input & Hidden_Direct & Direct_Out & Correct_Out \\
\hline $\mathrm{O}$ & & & & \\
\hline 100 & & & & \\
\hline 400 & & & & \\
\hline
\end{tabular}

Fig. 10. Snapshots of the activations in the Direct channel's layer for the selected processing cycles when a stimulus containing a pattern with missing parts is presented to the channel.

Pattern with missing parts: In figure 10, the simulation behaviour of the Direct channel is presented when a stimulus containing a pattern with missing parts is presented as input. The activation patterns of the Hidden_Direct and the Direct_Output layers in this simulation are very similar to those in the last simulation (Figure 9). This is because the Hidden_Direct layer discriminates among the patterns on the basis of the intensity values and generates the template of only those parts of the pattern in the last simulation which are visible in the missing pattern stimulus.

\begin{tabular}{|c|c|c|c|c|}
\hline Cycle & Input & Hidden_Direct & Direct_Out & Correct_Out \\
\hline o & & & & \\
\hline 100 & & & & \\
\hline 400 & & & & \\
\hline
\end{tabular}

Fig. 11. Snapshots of the activations in the Direct channel's layer for the selected processing cycles when a stimulus with occluded and occluding patterns having the same intensity value is presented to the network.

Occluded and occluding patterns having same intensity values: Figure 11 shows the processing results when a stimulus composed of occluded and occluding patterns having the same intensity values is presented to the channel. This time the Hidden_Direct layer generates a template that contains both the occluding as well as the occluded pattern (cycle: 100-400). The Direct_Output layer indicates three possible pattern classes (cycle: 100-400) for the occluded pattern with varying degree of confidence, depicted by different intensity values. 
This channel performs recognition of the patterns in a size, position and shape invariant manner. In this simulation it will perform processing independently of the other two channels. But, in the case of the integrated model, this channel receives early top-down cues from the Direct channel and interacts with the Spatial Channel. The same three stimuli were presented to this channel that were presented to the other two channels in the previous simulations.

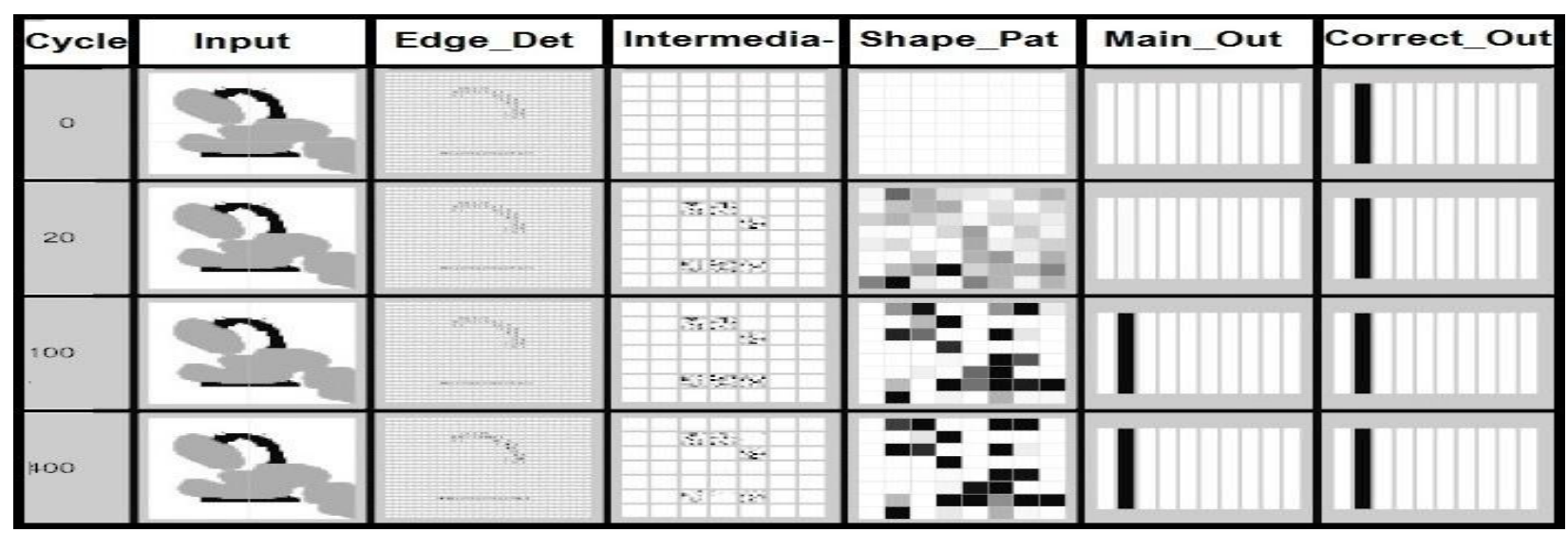

Fig. 12. Activations of the Main channels' layers when stimulus containing occluded and occluding pattern having different intensity values are presented to the channel. In this figure, in order from left to right, the columns represent: Number of processing cycles (how far computation of the activation has progressed), activations in the Input layer, the Edge_Rep layer, the Intermediate_Rep, the Shape_Pat and the Main_Output layer of the network. Dark colours denote high activation values, light colours low activation.

Occluded and occluding patterns having different intensity values:

Figure 12, shows the results of the simulation when a stimulus containing occluded and occluding patterns having the same intensity values was presented to the channel. It can be observed that, like the Hidden_Direct layer of the direct channel, the Edge_Rep layer in this channel differentiate the input patterns on the basis of the intensity values and encodes edges that belong to patterns with a specific intensity value (cycle: $0-400$ ), which in this case is the intensity value of the occluded pattern. In this simulation the main channel correctly recognizes the pattern identity (cycle: 100-400).

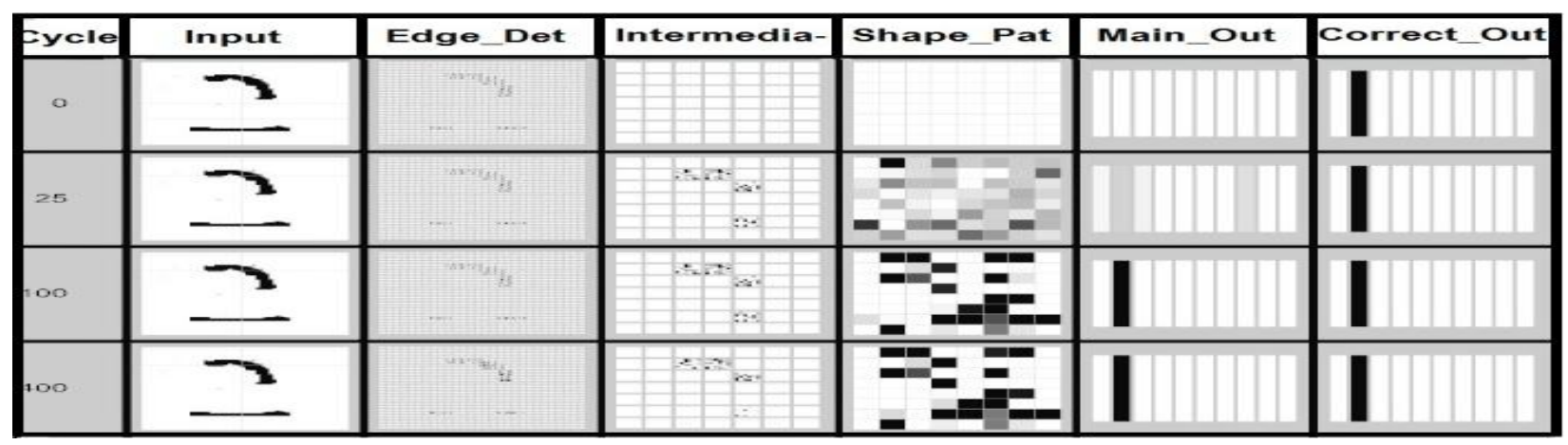

Fig. 13. Activations of the Main channels' layers for the selected processing cycles, when a stimulus containing patterns with missing parts is presented to the channel.

Pattern with missing parts: Figure 13, displays results when a stimulus containing patterns with missing parts was presented to the main channel. The processing behaviour of the channel in this simulation is very similar to its behaviour in the last simulation (Figure 12). In this simulation, the channel correctly recognizes the pattern class (cycle: 100-400). 


\begin{tabular}{|c|c|c|c|c|c|c|}
\hline Cycle & Input & Edge_Det & Intermedia- & Shape_Pat & Main_Out & Correct_Out \\
\hline 0 & & & & & & \\
\hline 15 & & & & 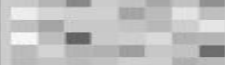 & & \\
\hline 100 & & & & $-x^{2}$ & & \\
\hline 50 & & & & 프 & & \\
\hline
\end{tabular}

Fig. 14. Activations of the Main channels' layers for selected processing cycles, when a stimulus with occluded and occluding patterns having the same intensity value is presented to the network.

Occluded and occluding patterns having the same intensity values: In this simulation, Figure 14, the Edge_Rep layer extracts edges from both the occluding as well as the occluded pattern (cycle: 0-400) as there is no way (bottom-up cues) to distinguish between the two patterns. In this particular case the main network could not recognize the patterns correctly (cycle: 15-400).

Simulation 3: Interactive pattern segmentation by the spatial channel

As stated earlier the main functionality of this channel is to segment the bottom up saliency map and develop a map for the occluded pattern. Here we will present the processing behaviour of this channel.

\begin{tabular}{|c|c|c|c|c|c|c|}
\hline Cycle & Input & BotUp_Sal & Temp_Patch & Recon_Pat & Spatial & Attention \\
\hline 60 & & & & -- & & \\
\hline 11 & & & - & 一 & & $-=$ \\
\hline 160 & & & $=$ & & & $=$ \\
\hline 300 & & & -1 & 5 & & III \\
\hline & & & & & & III \\
\hline
\end{tabular}

Fig. 15. Snapshots of the activations in the Spatial channel's layers for the selected processing cycles, when a stimulus containing occluded and occluding patterns having different intensity values are presented to the channel. In this figure, in the order from left to right, the columns represent: number of processing cycles, activations in the Input layer, the BotUp_Saliency, the Temp_Patch, the Recon_Pat, the Spatial_Output and the Attention layer of the channel.

Occluded pattern with noise having different intensity values: Consider Figure 15, for an illustration of this simulation. First of all, an occluded pattern is presented to this channel through the Input layer. Activation of the pattern spread to the BotUp_Saliency layer, which generates a saliency map or template of the input pattern (cycle: 60-400). Next, the Temp_Patch layer gets activation from the BotUp_Saliency layer and due to the low value of K (for the KWTA) a relatively small number of its units become active. This means activation of only a small portion of the saliency map in the previous layer. Due to self-connections among the units within the layer, the active units in this layer appear in the form of one or two connected patches (cycle: 60-400). This activation pattern in the Temp_Patch layer is temporal in nature, because due to 'accommodation' functionality of the units in this layer, presently active units will become tired after some time, and give way to other units of this layer to become active. The presently active pattern in the Temp_Patch layer will activate the most closely matched learned pattern class at 
the Spatial_Output layer. Meanwhile, the Temp_Patch layer transfers a copy of its activation pattern to the Recon_Pat layer, which unlike the Temp_Patch layer keeps the active pattern for long time (in this simulation it keeps the pattern for the whole trial duration or 400 cycles). The Recon_Saliency layer also sends class specific signals to the Spatial_Output layer. The Spatial_Output layer in return sends feedback to the Temp_Patch and Recon_Pat layers, and thus modulates activations in these two layers. This interactive processing proceeds in small steps and consequently groups of units at the Temp_Patch layer activates (moves) over the spatial area, which in this case represents the pattern ' 5 '. In the meantime, the Recon_Pat layer keeps traces of the ephemeral groups at the Temp_Pat layer (cycle: 60-400). As the processing cycle proceeds, the pattern at the Recon_Pat layer grows and sends increasingly strong signals to the Spatial_Output layer for pattern ' 5 ', as well as it more strongly interacts with the Temp_Patch layer. The resulting map at the Recon_Pat layer emerges in the form of a rough template representing the pattern ' 5 ' (cycle: 300-400). This template contains the spatial as well as the shape information of the pattern.

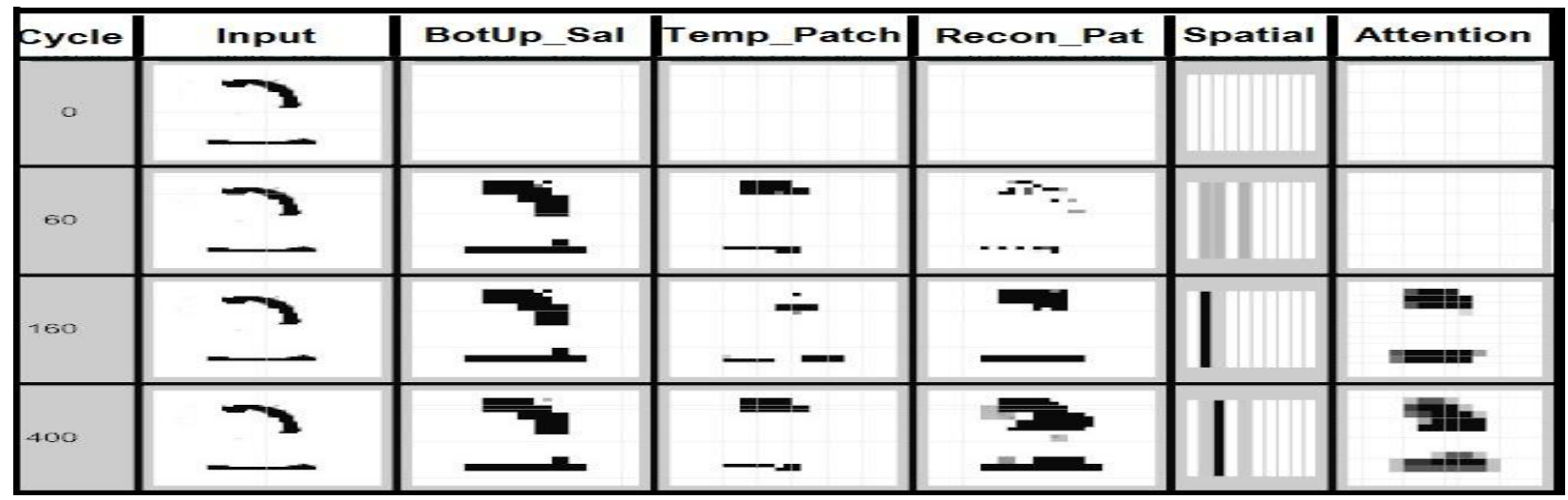

Fig. 16. Snapshots of the activations in the Spatial channel's layers when a stimulus having a pattern with missing parts is presented to the channel.

Pattern with missing parts: Figure 16, displays the activation patterns of the different layers for this simulation. The BotUp_Saliency layer generates the map of the visible parts of the pattern. The Temp_Patch layer activates some parts of the pattern and send signals to the Spatial_Output (cycle: 60) layer. The Spatial_Output layer identifies these patches as a part of pattern '2' and sends back the signal to the Temp_Patch layer in favour of pattern ' 2 '. The Temp_Patch layer gets top down signals in favour of pattern '2', but lacks the bottom up signal for this pattern because some parts of the pattern is missing. This makes the Temp_Patch layer activation unable to activate the units along the shape or spatial area of the pattern ' 2 '. Instead, under top down and bottom up pressure the Temp_Patch layer activates some patches that match the pattern ' 3 ' and accordingly classified at the Spatial_Output layer. The Spatial_Output layer send back top down signal for this pattern, but due to missing parts the shape of this pattern cannot be constructed. This activation dynamics cause a kind of confusion for the channel and it can be noted that the Recon_Pat layer cannot reconstructs a legible pattern (cycle: 400).

\begin{tabular}{|c|c|c|c|c|c|c|}
\hline Cycle & Input & Botup_sal & Temp_Patch & Recon_Pat & Spatial & Attention \\
\hline o & & & & & & \\
\hline & & & - & $\cdots$ & & \\
\hline 160 & & & & & & \\
\hline 400 & & & & & & \\
\hline
\end{tabular}

Fig. 17. Snapshots of the activations in the Spatial channel's layers when a stimulus containing occluded and occluding patterns having the same intensity values is presented to the channel.

Occluded and occluding pattern having the same intensity values: The behaviour of the channel for this stimulus is very similar to the channel behaviour in case of a stimulus containing occluded and occluding patterns having different intensity values. The reason is that the BotUp_saliency layer generates the same map in both cases. Figure 17, shows the activation patterns of the layers for this stimulation. 


\section{Simulation 4: The integrated model}

After presenting the simulation behaviour of the three individual channels, the simulation behaviour of the model as a whole will now be presented. First of all the same three stimuli will be presented to the model that were presented to the individual channels in the previous simulations. After that a couple of additional stimuli will be processed with the model for further illustration.

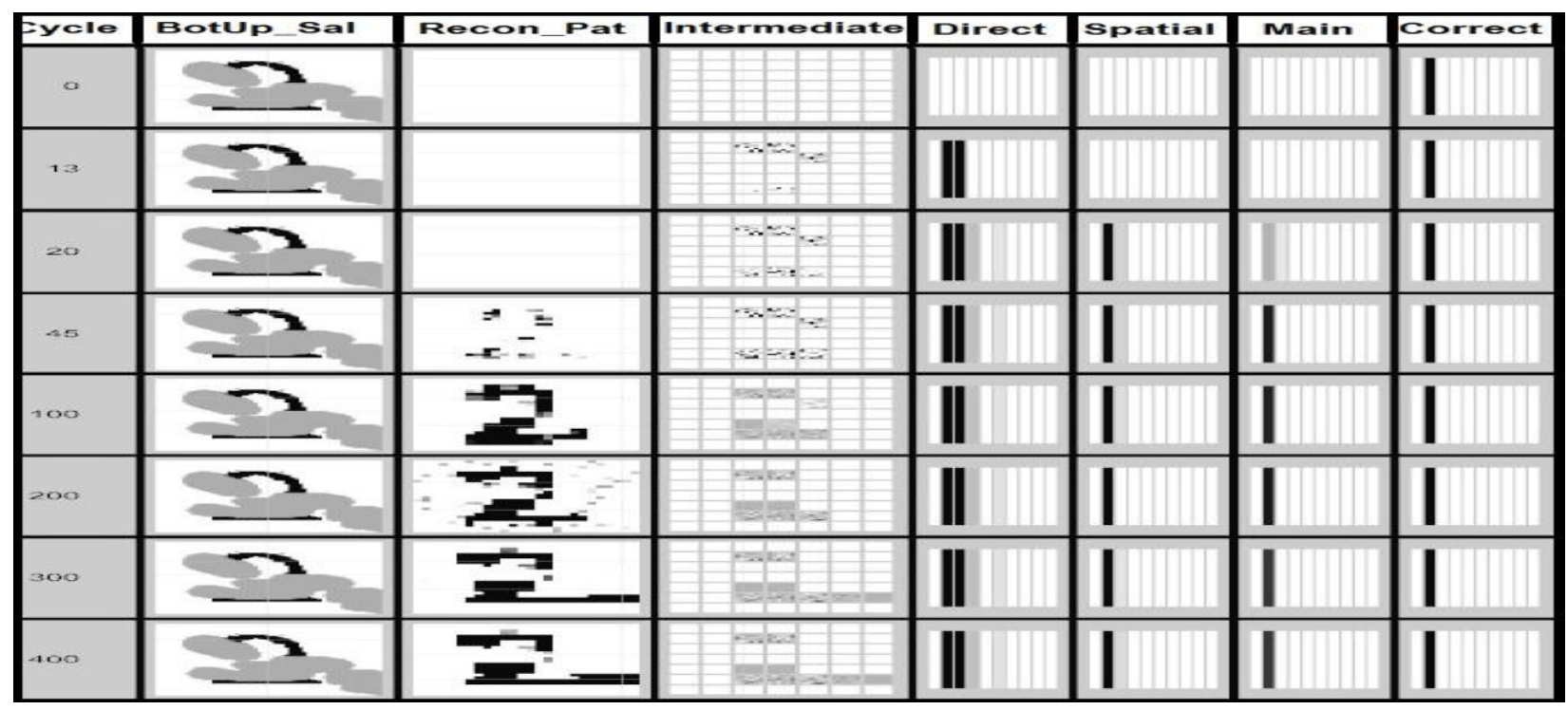

Fig. 18. Activations of the integrated model's layers for the selected processing cycles, when a stimulus containing occluded and occluding patterns having different intensity values are presented to the channel. In this figure, in the order from left to right, the columns represent: number of processing cycle, activations in the Input, the BotUp_Saliency, the Recon_Pat, the Intermediate_Rep, the Direct_Output, the Spatial_Output, the Main_Output and the Correct_Output layer.

Occluded and occluding patterns having different intensity values: Figure 18, shows the simulation behaviour of the model as the simulation process progresses. In response to the stimulus containing occluded patterns, the Output_ Direct layer responds by activating two of its units, representing patterns ' 2 ' and ' 3 ' (cycle: 13). The Direct_Output layer modulates the Spatial_Output and Main_Output layers, which in turn modulate the respective channels by propagating top down signals along their hierarchy. There is a slight difference in processing of the integrated model than that of the individual channels: In the integrated model, the Main and the Spatial channels get top down modulation before their respective output units get bottom up signals. Now, consider cycle: 45; the output layers of all three channels show the same result. The Recon_Pat layer constructs the shape of the pattern ' 2 ' under the influence of both top down as well as bottom-up cues. It can also be seen that the layer Edge_Rep gets modulated under the influence of the Spatial channel through the Attention layer (cycle: 100-400). The model correctly recognizes the occluded pattern in the stimulus as ' 2 ' (cycle: 100-400).

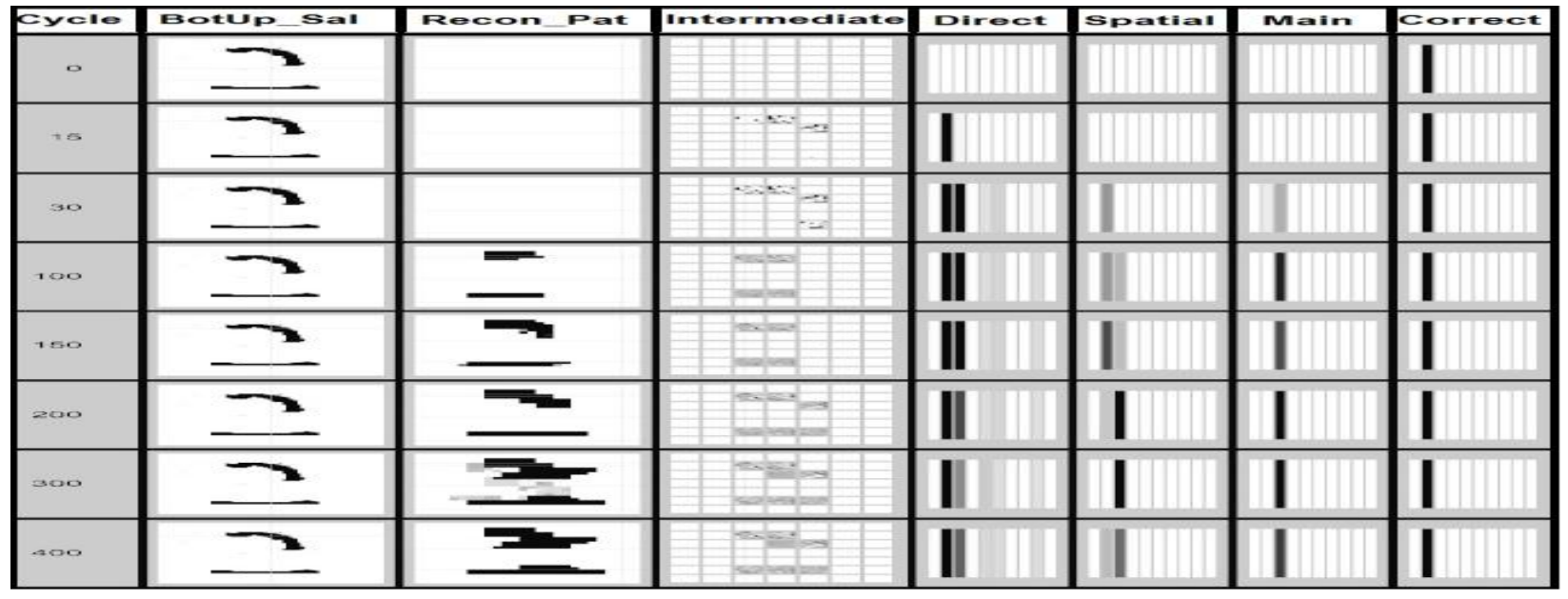


Fig. 19. Activations of the integrated model's layers for the selected processing cycles, when stimulus having patterns with missing parts are presented to the channel.

Patterns with missing parts: Figure 19, presents the simulation results when the stimulus is presented to the model. Initially, the Direct_Output layer categorises the pattern as either pattern '2' or ' 3 '. The Spatial_Output layer gets modulated in favour of pattern '2' and the Spatial channel tries to reconstruct the pattern. But, due to lack of bottom-up activations for this pattern, due to missing parts, the Spatial channel cannot construct it correctly and gets confused between pattern ' 2 ' and ' 3 '. Consequently, the Recon_Pat layer shows an incomplete pattern and its interaction with the main channel results in incorrect recognition of the pattern.

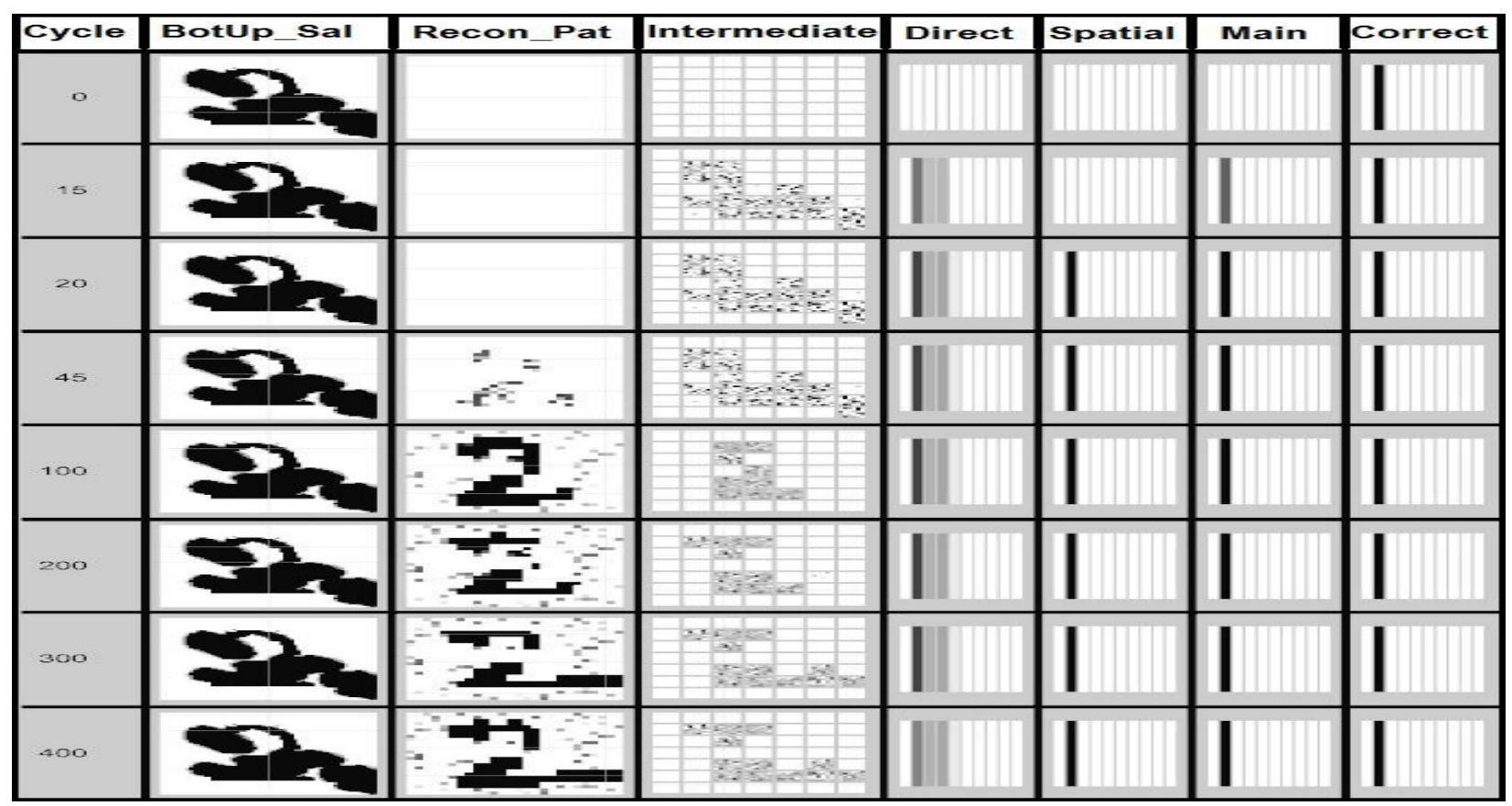

Fig. 20. Activations of the integrated model's layers for the selected processing cycles, when stimulus containing patterns with occluded and occluding patterns having the same intensity values is presented to the channel.

Occluded and occluding patterns having the same intensity values: Figure 20, shows the simulation results when the stimulus is presented to the model. The behaviour of the model is similar to the model behaviour in the previous simulation. The only difference is that the Direct_Output layer activates a unit, which represents pattern ' 2 ', more strongly than the others and under its influence the model correctly reconstructs and recognizes the occluding pattern in the input stimulus. 


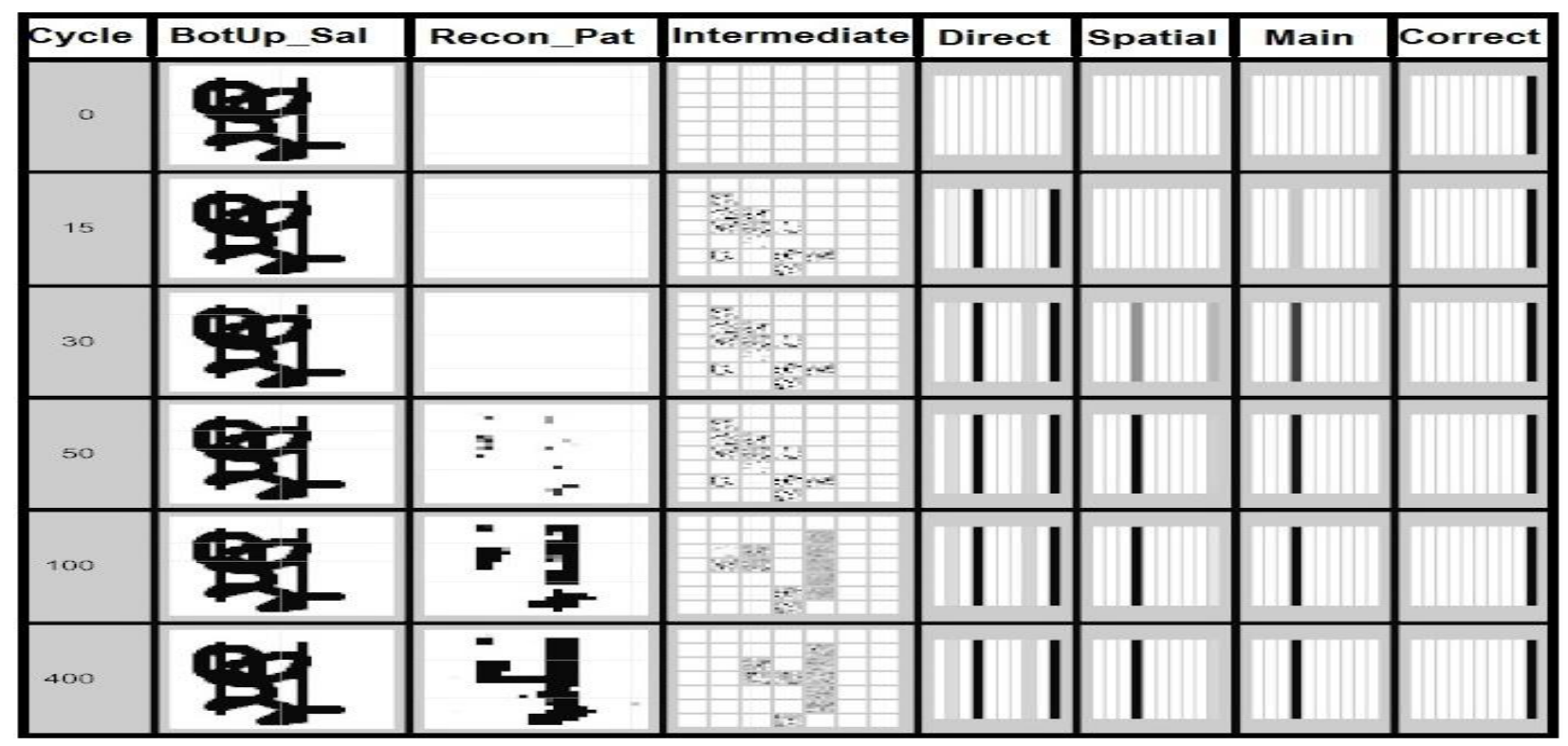

Fig. 21. Activations of the integrated model's layers for selected processing cycles.

Some more examples: In order to further illustrate the behaviour of the model, a couple of additional simulations will be presented.

Figure 21, presents the behaviour of the model when a stimulus, which contains the occluded pattern ' $\mathrm{H}$ ' is presented to the model. In this simulation the Direct channel classifies the occluded pattern as either ' $\mathrm{H}$ ' or ' 4 '. This is because pixels that forms pattern '4' are somewhat a subset of pixels forming pattern ' $H$ '. The initially active patches at the Temp_Patch layer match pattern '4' and thus the Spatial_Ouput layer recognizes this patch as a part of pattern ' 4 ' (cycle: 30-400). Consequently, pattern ' 4 ' is reconstructed (cycle: 50-400) and the occluded pattern is recognized as pattern ' 4 '.

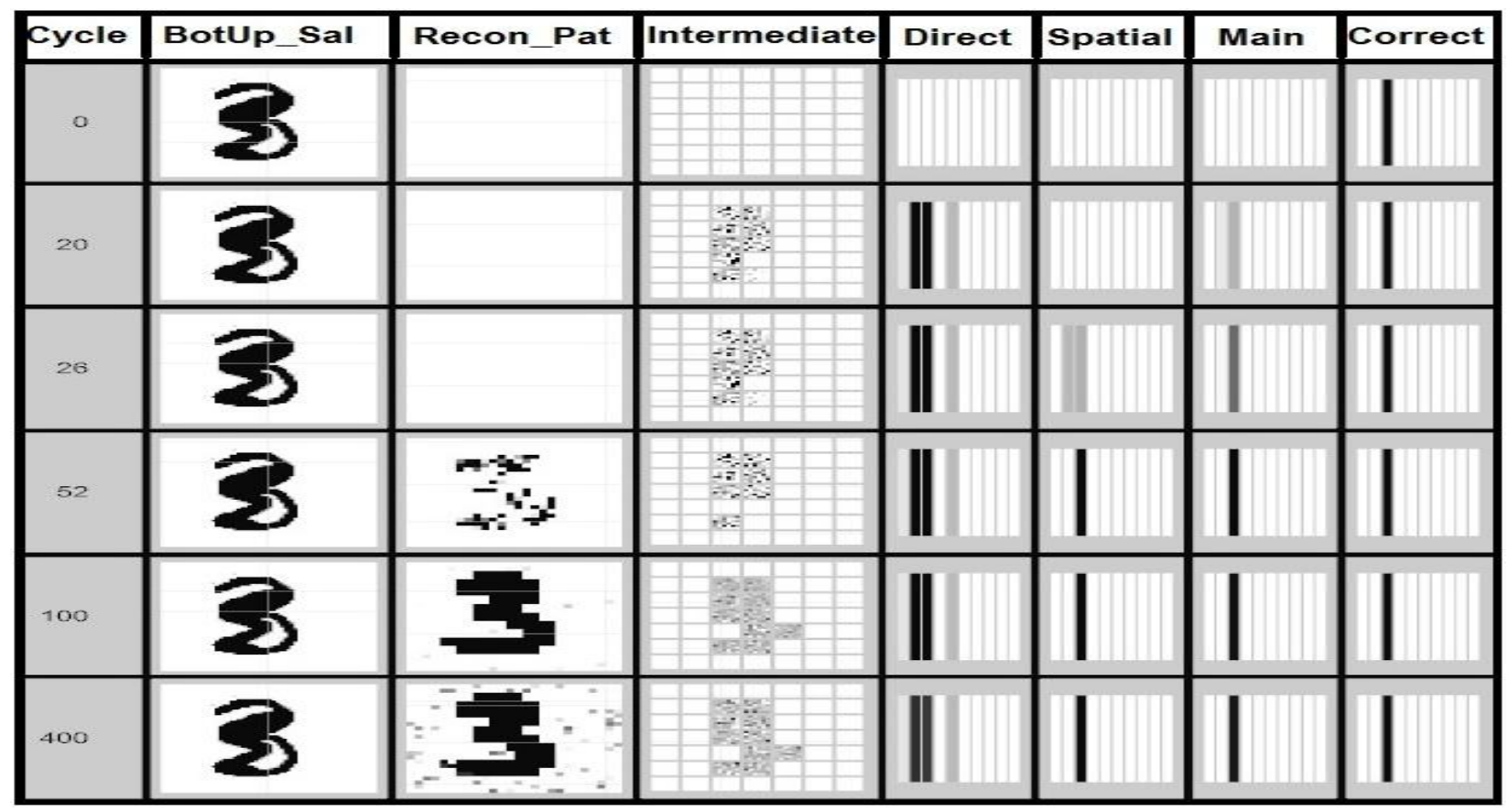

Fig. 22. Activations of the integrated model's layers for the selected processing cycles.

Figure 22, shows the model behaviour when a stimulus containing pattern ' 3 ' is presented. The model correctly reconstructs and recognizes the pattern. 


\section{Discussion and Conclusion:}

In this paper a biologically-inspired model for recognition of occluded patterns is presented. The model takes input stimuli containing occluded patterns and then segments and recognizes the occluded patterns in it. Here the segmentation and recognition processes are considered highly interdependent and proceed in parallel in small incremental steps. The simulation results indicate that the model provides a biologically-inspired solution to the problem. Moreover, the simulation behaviour of the model is in accordance with human behaviour on similar tasks.

An eye-tracking study was conducted to investigate the human behaviour on the recognition task of occluded patterns. The results of the experiment underline the importance of early top-down cues. Furthermore, the results suggest an interactive processing strategy for solving the task. Based on these findings an extended interactive processing approach is proposed. The extended interactive processing approach is in fact an interactive approach with a so called 'pre-figural', rapid recognition step which computes early top-down cues. These top-down cues provide an early identity guess for the occluded pattern to the model and, therefore facilitate the processing by avoiding any confusion in the beginning. It is important to note that the computation of early top-down cues in this model is different than the one proposed by Peterson and colleagues. According to their account the 'pre-figural step' takes place before any kind of figure-ground organization and on the basis of luminance contours. In our model the computation of top-down cues are based on template matching combined with bottom-up intensity based information, i.e., the pre-knowledge of the occluded pattern intensity values are used. In the same way, this approach is different from the interactive processing approach as the early top-down cues do not fit well with the basic idea of the interactive processing approach, which envisage the small incremental steps unlike the recognition of the template in a single step.

The main contribution of this work is how segmentation of those parts of the saliency maps, which represent occluded patterns in the input stimuli, is encoded. In this work we hypothesize that the Dorsal pathway of the visual cortex, in the human brain, encodes the shape information in addition to encoding the spatial features of the patterns in the input. This encoded information of the patterns shape is used as top-down cues to modulate processing along the pathway. The segmentation and somewhat reconstruction of the patterns' saliency maps is done as a result of interaction between the top-down pattern and bottom-up image-based cues. This approach is different from the other biologically-inspired approaches as in most other models the dorsal pathway is assumed to encode just spatial information of the input. This information is encoded, usually, by generating the saliency map on the basis of bottom-up cues. There are other approaches that uses top-down cues for the refinement of the saliency map in favour of one specific pattern but no approach uses shape information in the dorsal pathway. Moreover, in those processing models top-down modulation is performed in just one atomic step unlike the interactive modulation employed in our model.

Another contribution of this work is that it demonstrates the importance of direct connections from the lower to the higher levels along the information processing hierarchy of the model. There are numerous evidence of direct connections in the visual system of the humans. These connections have seldom been modelled in computational models for pattern recognition in the way we did in our three channel model. Here, we hypothesize and model these connections to compute early top-down cues. These cues help restrict the choices of processing along the two other processing pathways. For example, in the Spatial pathway of the model, an important and critical step is the emergence of the small patches which are fetched from the bottom-up saliency layer and send to the higher levels for classification. The selection of this patch (first time) is a random process and thus it can be from any part of the map which might be noise. In this situation there is always a chance that this patch will be recognized with some false identity and may cause confusion in the pathway. To avoid this situation the early top-down cues play an important role by providing the pathway an initial guess. This guess constrain the choices of the pathway in terms of making further selection of patches, as well as class assignment to the patches at the higher level. It is true that accuracy of the early guess is arbitrary but it is a far better alternative than not using it all. Nevertheless, this early guess is based on some systematic processes unlike random selection of the patch from the bottom-up saliency.

In the future, the model can be extended in several ways. One possible direction is to make the model work with more natural and colour images. It requires extension of the saliency map of the model. Though, on the one hand complex colour images would add complexity and difficulty for the model, on the other hand they will help in segmentation and the recognition process by providing colour cues. Another possible extension is to implement scale spaces in the model, allowing it to handle larger variations in the natural pattern sizes.

\section{Acknowledgements:}

The authors wish to thank IKT-studion for the use of, as well as help with the eye tracker.

\section{References:}

[1] M. A. Peterson, "Object recognition processes can and do operate before figure-ground organization," Current Directions in Psychological Science, vol. 3, no. 4, pp. 105-111, 1994.

[2] S. P. Vecera and R. C. O'reilly, "Figure-ground organization and object recognition processes: An interactive account.," Journal of Experimental Psychology: Human Perception and Performance, vol. 24, no. 2, p. 441, 1998.

[3] A. Needham, "Object Recognition and Object Segregation in 4.5-Month-Old Infants," Journal of experimental child psychology, vol. 78, no. 1, pp. 3-24, 2001.

[4] R. Bajcsy, F. Solina, and A. Gupta, "Segmentation versus object representation-are they separable?," 1990.

[5] I. Biederman, "Recognition-by-components: A theory of human image understanding.," Psychological review, vol. 94, no. 2, p. 115, 1987. 
[6] S. M. Kosslyn, "Seeing and imagining in the cerebral hemispheres: A computational approach.," Psychological review, vol. 94, no. 2, p. 148, 1987.

[7] D. Marr, "Early processing of visual information.," Philosophical transactions of the Royal Society of London. Series B: Biological sciences, vol. 275, no. 942, pp. 483-519, 1976.

[8] U. Neisser, Cognitive psychology. Appleton-Century-Crofts New York, 1967.

[9] E. Rubin, "Figure and ground," Readings in perception, pp. 194-203, 1958.

[10] M. Wertheimer, "Principles of perceptual organization," Readings in perception, pp. 115-135, 1958.

[11] M. A. Peterson and B. S. Gibson, "The initial identification of figure-ground relationships: Contributions from shape recognition processes," Bulletin of the Psychonomic Society, vol. 29, no. 3, pp. 199-202, 1991.

[12] M. A. Peterson and B. S. Gibson, "Shape recognition inputs to figure-ground organization in three-dimensional grounds.," Cognitive Psychology, 1993.

[13] M. A. Peterson and B. S. Gibson, "Must figure-ground organization precede object recognition? An assumption in peril," Psychological Science, vol. 5, no. 5, p. 253, 1994.

[14] M. A. Peterson and B. S. Gibson, "Object recognition contributions to figure-ground organization: Operations on outlines and subjective contours," Attention, Perception, \& Psychophysics, vol. 56, no. 5, pp. 551-564, 1994.

[15] E. Borenstein and S. Ullman, "Class-specific, top-down segmentation," Computer Vision-ECCV 2002, pp. 639-641, 2002.

[16] Z. Tu, X. Chen, A. L. Yuille, and S. C. Zhu, "Image parsing: Unifying segmentation, detection, and recognition," International Journal of Computer Vision, vol. 63, no. 2, pp. 113-140, 2005.

[17] V. Ferrari, T. Tuytelaars, and L. Van Gool, "Simultaneous object recognition and segmentation by image exploration," Computer VisionECCV 2004, pp. 40-54, 2004

[18] S. P. Vecera and M. J. Farah, "Is visual image segmentation a bottom-up or an interactive process?," Attention, Perception, \& Psychophysics, vol. 59, no. 8, pp. 1280-1296, 1997.

[19] G. M. Reicher, "Perceptual recognition as a function of meaningfulness of stimulus material.," Journal of experimental psychology, vol. 81 , no. 2, p. 275, 1969.

[20] D. D. Wheeler, "Processes in word recognition," Cognitive Psychology, vol. 1, no. 1, pp. 59-85, 1970

[21] W. Prinzmetal and M. Millis-Wright, "Cognitive and linguistic factors affect visual feature integration," Cognitive Psychology, vol. 16, no. 3, pp. 305-340, 1984.

[22] S. Palmer and I. Rock, "Rethinking perceptual organization: The role of uniform connectedness," Psychonomic Bulletin \& Review, vol. 1, no. 1, pp. 29-55, 1994.

[23] S. Ullman, "Aligning pictorial descriptions: An approach to object recognition," Cognition, vol. 32, no. 3, pp. 193-254, 1989.

[24] M. C. Mozer, R. S. Zemel, M. Behrmann, and C. K. I. Williams, "Learning to segment images using dynamic feature binding," Neural Computation, vol. 4, no. 5, pp. 650-665, 1992.

[25] M. C. Mozer, R. S. Zemel, M. Behrmann, and C. K. I. Williams, "Learning to segment images using dynamic feature binding," Neural Computation, vol. 4, no. 5, pp. 650-665, 1992.

[26] I. Rock and B. Campbell, An introduction to perception. Macmillan New York, 1975.

[27] J. L. McClelland, "Toward a theory of information processing in graded, random, and interactive networks.," 1993.

[28] D. E. Rumelhart, "The architecture of mind: A connectionist approach," Mind readings, pp. 207-238, 1998.

[29] A. R. Weeks and G. E. Hague, "Color segmentation in the HSI color space using the K-means algorithm," in Proceedings of SPIE, 1997, vol. 3026, pp. 143-154.

[30] J. Du Buf, M. Kardan, and M. Spann, “Texture feature performance for image segmentation,” Pattern recognition, vol. 23, no. 3, pp. 291-309, 1990.

[31] U. Montanari, "On the optimal detection of curves in noisy pictures," Communications of the ACM, vol. 14, no. 5, pp. 335-345, 1971.

[32] E. Borenstein and S. Ullman, "Combined top-down/bottom-up segmentation," IEEE Transactions on Pattern Analysis and Machine Intelligence, vol. 30, no. 12, pp. 2109-2125, 2008

[33] B. Leibe, A. Leonardis, and B. Schiele, "Robust object detection with interleaved categorization and segmentation," International Journal of Computer Vision, vol. 77, no. 1-3, pp. 259-289, 2008

[34] I. Biederman, "Perceiving real-world scenes," Science, vol. 177, no. 4043, pp. 77-80, 1972.

[35] M. C. Potter, "Meaning in visual search," Science, vol. 187, no. 4180, pp. 565-566, 1975.

[36] S. Thorpe, D. Fize, C. Marlot, and others, "Speed of processing in the human visual system," nature, vol. 381, no. 6582, pp. 520-522, 1996.

[37] F. Kelly and S. Grossberg, "Neural dynamics of 3-D surface perception: Figure-ground separation and lightness perception," Attention, Perception, \& Psychophysics, vol. 62, no. 8, pp. 1596-1618, 2000.

[38] M. Saifullah and R. Kovordányi, "Emergence of attention focus in a biologically-based bidirectionally-connected hierarchical network," Adaptive and Natural Computing Algorithms, pp. 200-209, 2011.

[39] R. C. O'Reilly and Y. Munakata, Computational explorations in cognitive neuroscience: Understanding the mind by simulating the brain. The MIT Press, 2000.

[40] S. R. Lehky and A. B. Sereno, "Comparison of shape encoding in primate dorsal and ventral visual pathways," Journal of neurophysiology, vol. 97, no. 1, p. 307, 2007.

[41] A. Sereno and J. Maunsell, "Shape selectivity in primate lateral intraparietal cortex," J. Exp. Psychol. Hum. Percept. Perform, vol. 12, pp. 388-391, 1987.

[42] B. Aisa, B. Mingus, and R. O’Reilly, "The emergent neural modeling system," Neural networks, vol. 21, no. 8, pp. 1146-1152, 2008.

[43] R. C. O'reilly, "Biologically plausible error-driven learning using local activation differences: The generalized recirculation algorithm," Neural computation, vol. 8, no. 5, pp. 895-938, 1996. 